\title{
The Solar Flare: A Strongly Turbulent Particle Accelerator
}

\author{
Loukas Vlahos $^{1}$, Sam Krucker ${ }^{2}$ and Peter Cargill ${ }^{3,4}$ \\ 1 Department of Physics, University of Thessaloniki, 54124 Thessaloniki, Greece \\ vlahos@astro.auth.gr \\ 2 Space Physics Research Group, University of California, Berkeley, USA \\ krucker@apollo.ssl. berkeley.edu \\ 3 Space and Atmospheric Physics, The Blackett Laboratory, Imperial College, \\ London SW7 2BW, UK \\ 4 School of Mathematics and Statistics, University of St Andrews, St Andrews, \\ KY16 9SS UK p.cargill@imperial.ac.uk
}

\section{Introduction}

The topics of explosive magnetic energy release on a large scale (a solar flare) and particle acceleration during such an event are rarely discussed together in the same article. Many discussions of magnetohydrodynamic (MHD) modelling of solar flares and/or CMEs have appeared (see [143] and references therein) and usually address large-scale destabilisation of the coronal magnetic field. Particle acceleration in solar flares has also been discussed extensively $[74,164,116,166,87,168,95,122,35]$ with the main emphasis being on the actual mechanisms for acceleration (e.g. shocks, turbulence, DC electric fields) rather than the global magnetic context in which the acceleration takes place.

In MHD studies the topic of particle acceleration is often presented as an additional complication to be addressed by future studies due to: (a) its inherent complexity as a scientific problem and (b) the difficulty in reconciling the large MHD and small (kinetic) acceleration spatial and temporal scales. The former point leads to the consideration of acceleration within a framework of simple plasma and magnetic field configurations, with inclusion of the complex magnetic field structures present in the real corona being often deemed intractible. For example, it is often assumed that large monolithic current sheets appear when an eruption drives simultaneously a CME and a

flare. The connection of such topologies with the extremely efficient transfer of magnetic energy to high energy particles remain an open question. The latter point is best seen by noting that models of energy release and acceleration requires methods that can handle simultaneously the large-scale magnetic field structures $\left(\sim 10^{4} \mathrm{~km}\right)$ evolving slowly (over the course of hours and days) 
and the small-scale dissipation regions $(\leq \mathrm{km})$ that evolve extremely rapidly (seconds to minutes).

The issues are well summarised in [143] where it is stated that "In future, we hope for a closer link between the macroscopic MHD of the flare and the microscopic plasma physics of particle acceleration. The global environment for particle acceleration is created by $M H D$, but there is a feedback, with the MHD affected by the nature of the turbulent transport coefficients". We draw attention in particular to the word "feedback": the fundamental question which needs to be fully addressed is the following: can we disengage the macroscopic MHD physics from the microscopic plasma physics responsible for particle acceleration? Current observational and theoretical developments suggest that for the case of explosive energy release in the solar atmosphere, such a separation is not possible.

The extraordinary efficiency of converting magnetic energy to energetic particles during solar flares (almost $50 \%$ of the dissipated magnetic energy will go into energetic particles, see Sect. 2), raises questions about the use of macroscopic (ideal or resistive MHD) theories as the description of impulsive energy release. The nonlinear coupling of large and small scales is extremely difficult to handle just by the use of transport coefficients. This is a problem which extends beyond solar physics and is one reason that our progress in understanding solar flares has been relatively slow over the last hundred years [34]. The overall goal of this paper is to show how alternative approaches to the "flare problem" can begin to show how the integration of large-scale magnetic field dynamics with particle acceleration processes is possible.

In this review we present a radically different approach, used less in the current literature, that connects the impulsive energy release in the corona with the complexity imposed in active regions by the turbulent photospheric driver [165]. The flare problem is thus posed differently, since it emerges naturally from the evolution of a complex active region. The convection zone actively participates in the formation and evolution of large scale structures by rearranging the position of the emerged magnetic field lines. At the same time the emergence of new magnetic flux rearranges the existing magnetic topologies in complex ways. 3-D magnetic topologies are thus constantly forced away from a potential state (if they were ever in one at all) due to slow (or abrupt) changes in the convection zone. Within these stressed large-scale magnetic topologies, localized short-lived magnetic discontinuities (current sheets) form spontaneously, and dissipate the excess energy in the form of small or large scale structures (nanoflares and flares/CMEs). We stress that the concept of the sudden formation of a distribution of unstable discontinuities inside a well organized large-scale topology is relatively new in the modeling of the solar flare phenomenon (see for example $[137,128]$ for important steps in the development of this approach).

The scenario of spatially distributed self-similar current sheets with localized dissipation evolving intermittently in time is supported by observations which indicate that flares and intense particle acceleration are associ- 
ated with fragmented energy dissipation regions inside the global magnetic topology $[168,25]$. There is strong evidence that narrow-band milli-second spike-emission in the radio range is directly associated with the primary energy release. Such emission is fragmented in space and time, as seen in radiospectrograms and in spatially resolved observations [173]. It can then be suggested that the energy release process is also fragmented in space and time, to at least the same degree as the radio spike-emission [24]. Also type III burst radio-emission, caused by electron beams escaping from flaring regions, appear in clusters, suggesting that fragmentation is a strong characteristic of the flaring region [23].

One approach which is able to capture the full extent of this interplay of highly localized dissipation in a well-behaved large scale topology ('sporadic flaring') is a special class of models $[109,110,167,115,81,82]$ which implement the concept of Self-Organized Criticality (SOC), proposed initially by Bak et al. [19]. The main idea is that active regions evolve smoothly until at some point(s) inside the large scale structure magnetic discontinuities (of all sizes) are formed and the currents associated with them reach a critical threshold. This causes a fast rearrangement of the local magnetic topology and the release of excess magnetic energy at the unstable point(s). This rearrangement may in turn cause a lack of stability in the immediate neighborhood, and so on, leading to the appearance of flares (avalanches) of all sizes that follow a well defined statistical law which agrees remarkably well with the observed flare statistics [38].

Based on the current observational and theoretical evidence discussed in this review, we suggest that our inability to describe properly the coupling between the MHD evolution and the kinetic plasma aspects of a driven flaring region is the main reason behind our lack of understanding of the mechanism(s) which causes flares and the acceleration of high energy particles. Let us now define the 'acceleration problem' during explosive energy release in the sun: We need to understand the mechanism(s) which transfer more than $50 \%$ of magnetic energy to large numbers $\left(10^{39}\right.$ particles in total) of energetic electrons and ions, to energies in the highly relativistic regime $(>100$ $\mathrm{MeV}$ for electrons and tens of GeVs for ions) on a short time scale (seconds or minutes), with specific energy-spectra for the different isotopes and charge states.

In Section 2 we briefly describe the key observational constrains. In Section 3 we present a brief overview of the main theories for impulsive magnetic energy release and in Section 4 we concentrate on the mechanisms on particle acceleration inside a more realistic and complex magnetic topologies. Finally in in Section 5 we discuss the ability of the proposed accelerators to explain the main observational results and in Section 6 we report the main points stressed in this review. 


\section{Observational Constraints}

\subsection{X-rays observations: diagnostics of energetic electrons and thermal plasmas}

Energetic electrons produce X-ray emission by collisions (the radiation mechanism responsible for the emission is non-thermal bremsstrahlung). The denser the plasma, the more collisions, and the more X-rays are produced (see Fig. 1 ). Therefore, X-rays produced by non-thermal electrons are strongest from the chromospheric footpoints of loops where the density increases rapidly. Indeed as the energetic electrons move into the chromosphere, they eventually lose all their energy through collisions. This scenario is usually called the "thick-target model" [30]. X-ray bremsstrahlung emission is in principle also emitted in the corona but the lower density there $\left(\sim 10^{9}\right.$ particles $\left./ \mathrm{cm}^{3}\right)$ is not big enough to stop energetic electrons or indeed to make them lose a significant amount of their energy ("thin-target model"). The estimated mean free path of an electron in the corona is $>10^{5} \mathrm{~km}$. In general present day instrumentation does not have a high enough signal to noise ratio to detect faint thin-target bremsstrahlung emission from the corona next to much brighter footpoints.
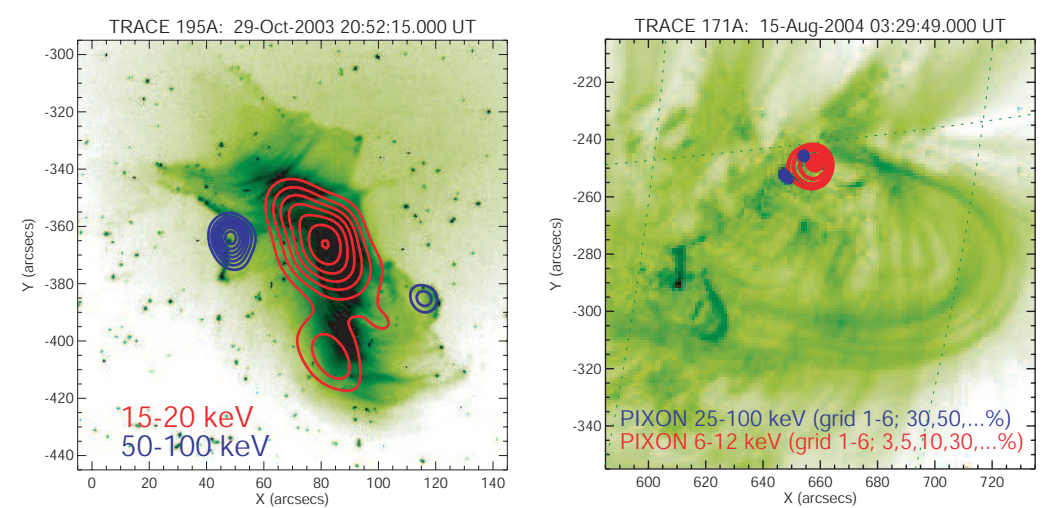

Fig. 1. Two examples of X-ray imaging in solar flares: (left) a large flare near disk center, (right) a small compact flare. Thermal emission in X-rays is shown by red contours, while non-thermal emission is shown as blue. The green images show EUV emission observed by TRACE with dark colors corresponding to enhanced intensity.

Thermal plasmas with temperatures above $1 \mathrm{MK}$ also radiate in X-rays by collisions (thermal bremsstrahlung). Thermal X-ray spectra have a steeply falling continuum component plus some line emissions. In solar flares, thermal emission generally dominates the X-ray spectrum below 10-30 keV. At higher energies, the flare spectra are generally flatter, having power laws with indices 
between 3-5, sometimes with breaks (see Fig. 2). This is the non-thermal bremsstrahlung component produced by energetic electrons.
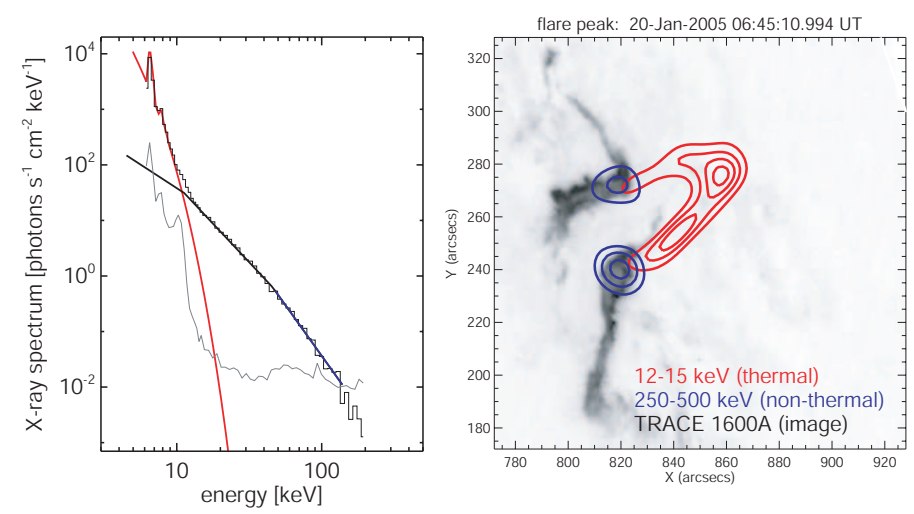

Fig. 2. Spectroscopy and imaging in X-rays: (left) a spatially integrated X-ray spectrum with a thermal fit in red and a broken power law fit (non-thermal emission) in blue. The data is shown in black and the instrumental background emission is shown in grey. (right) X-ray imaging with thermal emission in red and non-thermal in blue.

\subsection{Energy estimates}

Spectral X-ray observations provide quantitative estimates of the energy content. The non-thermal energy (i.e. the energy in the accelerated energetic electrons) can be estimated by inverting the photon spectrum to get the electron spectrum. The total energy is then derived by integrating the electron spectrum above a cutoff energy. The largest uncertainties in this derivation are due to the not-well-known cutoff energy. Often only an upper limit is known, giving lower limits to the non-thermal energy. Current estimates suggest that almost $50 \%$ of the total flare energy is deposited in energetic particles [56, 57].

Thermal flare energies are derived by fitting the thermal part of the X-ray spectra with a single temperature model, thus providing estimates of temperature and emission measure $E M \sim n^{2} V$. Here $V$ is an estimate of the volume occupied by the thermal plasma, usually obtained from images. From the emission measure and the volume, the number of heated electrons can be determined, each of which contains $1.5 \mathrm{kT} \mathrm{J}$. Assuming the same number of ions are heated, the total thermal energy becomes $3 k T \sqrt{E M / V}$. This energy estimate is equal to the total energy needed to obtain the observed heated flare plasma and does not account for radiative and conductive losses. The derived energies are therefore only lower limits.

In solar flares, the thermal and non-thermal energy estimates are generally correlated and are often the same order of magnitude. This is consistent with 
the picture that flare energy release first accelerates electrons which later lose their energy by collisions, heating chromospheric plasma (see [149] for recent results and references therein).

\subsection{Temporal correlation}

\section{Neupert effect}

If the flare-accelerated energetic electrons indeed heat the flare plasma, the $\mathrm{X}$-ray time profile of the thermal and non-thermal emission should reflect this: the non-thermal X-ray time profile should be a rough measure of how much energy is released in non-thermal electrons, and this is then the energy available for heating. So the larger the non-thermal X-ray flux, the more heating is expected. The time history of the integrated non-thermal X-ray flux roughly corresponds to the time profile of the thermal X-ray emission (this is called Neupert effect: see [162] for recent results).

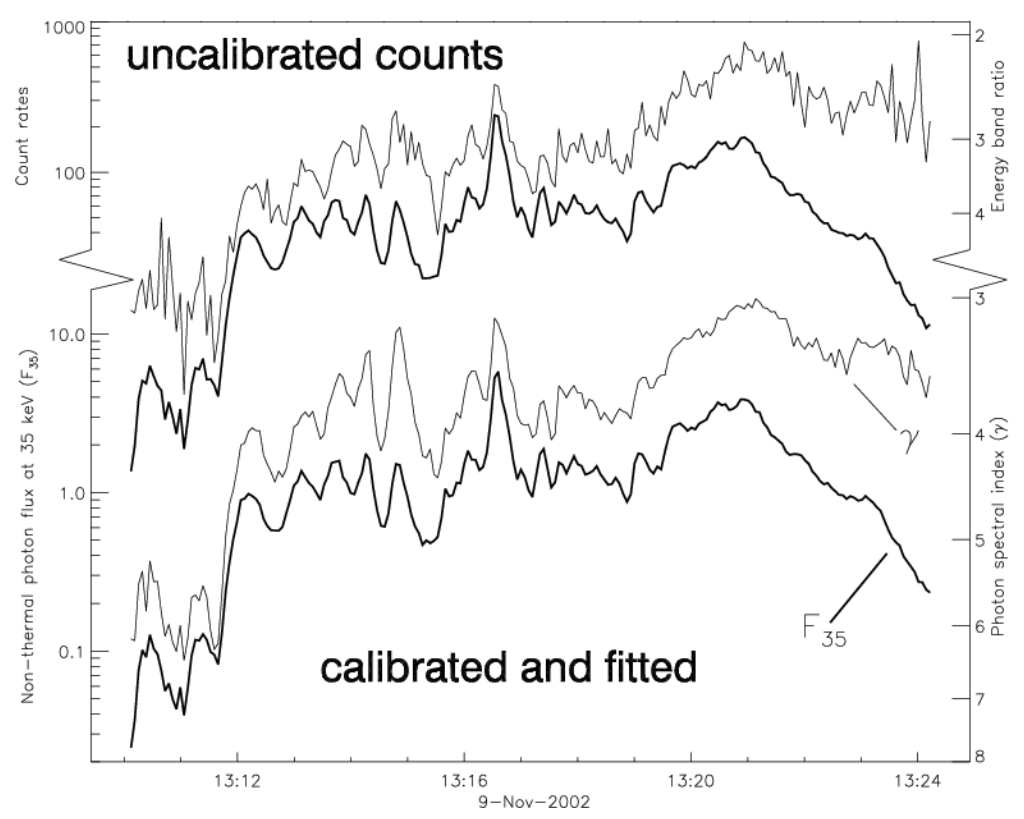

Fig. 3. Top: The spectral index (thin line) and flux (thick line) obtained from the uncalibrated total count rates flux in the energy bands $26-35 \mathrm{keV}$ and $35-44 \mathrm{keV}$ and their ratio. Bottom: the spectral index $\gamma$ (thin line) and non-thermal flux $F_{35}$ at $35 \mathrm{keV}$ in photons $\mathrm{s}^{-1} \mathrm{~cm}^{-2} \mathrm{keV}^{-1}$ (thick line) for the event of 9 November 2002, obtained by spectral fitting [70]. 


\section{Spectral evolution: soft-hard-soft}

A very strong temporal correlation is observed between the non-thermal Xray flux and the power law index of the photon spectrum: for each individual peak in the time series, the spectral shape hardens (flatter spectrum) until the peak and then softens (steeper spectrum) again during the decay (Fig. $3)$. This is referred to as the soft-hard-soft effect $[70,71]$ and seems to be a specific characteristic of the acceleration process. It is not understood. In some flares, the spectral behavior is different showing a gradual hardening during rise, peak, and decay for each individual burst. These events tend to be large and have a very good correlation with flares related to solar energetic particle (SEP) events [86]. Their behavior is also not understood. Note that spectral hardening can occur if electrons are trapped and low energy particles are lost first.

\subsection{Location of energy release}

\section{Coronal Hard X-ray (HXR) sources}

Coronal X-ray emission is most often from hot thermal loops as described above. However, for some events an additional X-ray source is observed originating above the thermal X-ray loops [112], called an 'above the loop top source' (ALT). First observed by Yohkoh, and only seen in a few flares (see Fig. 4), these sources are generally fainter with a softer spectrum than X-ray footpoints sources. There is no agreed interpretation of them at this time. If the footpoints and the ALT source are all produced by the same population of energetic electrons, the location of the ALT source indicates that the acceleration does not happen inside the flare loop. Therefore, it is generally speculated that the acceleration occurs above that loop. The relatively small number of flares with ALT sources might be because of the limited dynamic range of the observations alluded to earlier.

Several events do not follow this simple picture and the source is more complicated (see Fig. 5). RHESSI observations show several clear examples of ALT sources: the time evolution of these sources shows fast variations with several peaks of tens of second duration. The observed spectra are rather soft with power law indices around 5 and are better represented by non-thermal (power law) spectra than by thermal fits, although multi-thermal fits with temperatures up to $100 \mathrm{MK}$ can represent the data almost as well. The fast time variations are very difficult to explain for a thermal interpretation (i.e. repeated heating to $100 \mathrm{MK}$ and cooling on the same time scale). However, there are also difficulties with the non-thermal interpretation: the HXR producing electrons should significantly heat the ALT, but the hot thermal loops are observed below it. 

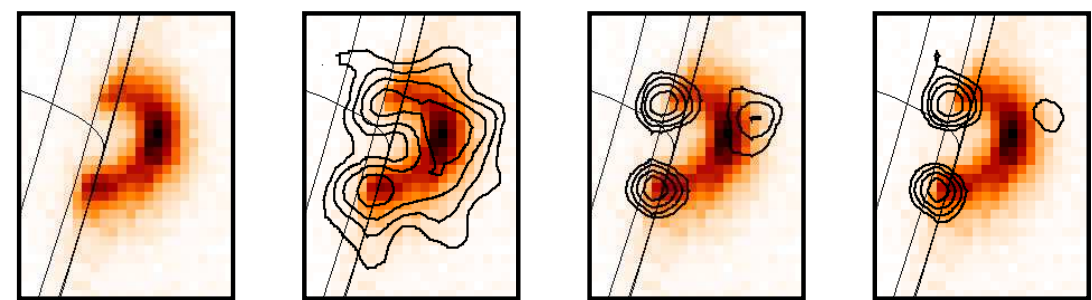

Fig. 4. Hard X-ray and soft X-ray images of the 13 January 1992 flare. The leftmost panel shows a soft X-ray image taken with the Yohkoh/SXT Be filter at 17:28:07 UT. From left to right, the remaining three panels show images contours at 14-23, 23-33, and 33-53 keV, respectively, taken from 17:27:35 - 17:28:15 UT by Yohkoh/HXT, overlaid on the same soft X-ray image. The contour levels are 6.25, 12.5, 25.0 and $50 \%$ of the peak value. The field of view is $59^{\prime \prime} \times 79^{\prime \prime}$ for all panels.

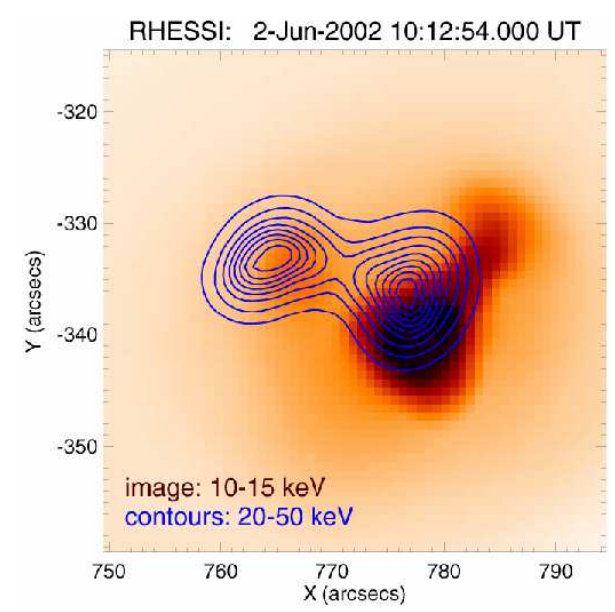

Fig. 5. X-ray imaging of a complex flare: the image (red) shows the thermal emission as seen by RHESSI at $10-15 \mathrm{keV}$, and the non-thermal emission is again given by blue contours

\section{Time of flight}

Further support for acceleration above the thermal flare loops is provided by timing studies of HXR footpoints at different energies. If energetic electrons at different energies are accelerated almost simultaneously at the same location, time of flight effects from the acceleration site to the HXR footpoints should be observed. This is indeed observed and it allows one to estimate the path length from the acceleration site to the HXR footpoints. The derived path lengths are generally longer than half the length of the flare loops connecting the HXR footpoints [16]. Although the error bars are large, this again suggests that the acceleration occurred above the flare loops. 


\section{Temperature structure}

Recent RHESSI observations also show support for particle acceleration above the main flare loop [157]. Evidence was found for a temperature gradient with decreasing temperatures from the possible coronal acceleration site towards lower and higher altitudes [156]. The hottest flare loops are expected to be the newly reconnected loops at largest altitude. Previously heated flare loops are at lower altitude and have already partly cooled down. For energetic electrons released upwards, the opposite is expected with the hottest emission at lowest altitude, as observed. Another explanation could be that there is direct heating at the particle acceleration site (as a by-product of the main acceleration process) that would produce a similar temperature profile.

\subsection{Footpoint motions}

Standard magnetic reconnection models predict increasing separation of the footpoints during the flare [143] as longer and larger loops are produced. If the reconnection process results in accelerated electrons [131], the HXR footpoints should show this motion. The motion is only apparent; it is due to the HXR emission shifting to footpoints of neighboring newly reconnected field lines. Hence, the speed of footpoint separation reflects the rate of magnetic reconnection and should be roughly proportional to the total HXR emission from the footpoints. Sakao, Kosugi, \& Masuda [150] analyzed footpoint motions in 14 flares observed by Yohkoh HXT, but did not find a clear correlation between the footpoint separation speed and the HXR flux. Recently, however, source motion seen in $\mathrm{H} \alpha$ was studied by Qiu et al. [146]. They found some correlation with HXR flux during the main peak, but not before or after.

RHESSI results $[59,91,92,71]$ show systematic but more complex footpoint motions than a simple flare model would predict. Krucker, Hurford, \& Lin [93] analyzed HXR footpoint motions in the July 23, 2002 flare (GOES X4.8-class). Above $30 \mathrm{keV}$, at least $3 \mathrm{HXR}$ sources are observed during the impulsive phase that can be identified with footpoints of coronal magnetic loops that form an arcade. On the northern ribbon of this arcade, a source is seen that moves systematically along the ribbon for more than 10 minutes. On the other ribbon, at least two sources are seen that do not seem to move systematically for longer than half a minute, with different sources dominating at different times. The northern source motions are fast during times of strong HXR flux, but almost absent during periods with low HXR emission. This is consistent with magnetic reconnection if a higher rate of reconnection (resulting in a higher footpoint speed) produces more energetic electrons per unit time and therefore more HXR emission. The absence of footpoint motion in one ribbon is inconsistent with simple reconnection models, but can be explained if the magnetic configuration is more complex. Also the motion of the northern footpoint is rather along the ribbon, contrary the perpendicular 
motions predicted by simple reconnection models. In some events the motion during the whole flare is clearly along the ribbons [71].

\subsection{Gamma Rays (emission above $>300 \mathrm{keV}$ )}

\section{Electron bremsstrahlung}

The non-thermal electron bremsstrahlung component can extend up to and above $10 \mathrm{MeV}$. This component is produced in the same way as the emission seen above $20 \mathrm{keV}$, but from electrons with higher energies. Generally the spectrum shows a hardening (flatter spectrum) above 0.5-1 MeV. Because the spectrum decreases steeply with energy, electron bremsstrahlung in the gamma-ray range is only observed for very large flares. Rarely, however, is it the dominant emission in the gamma-ray range. For most gamma ray flares, emission produced by energetic ions is present as well.

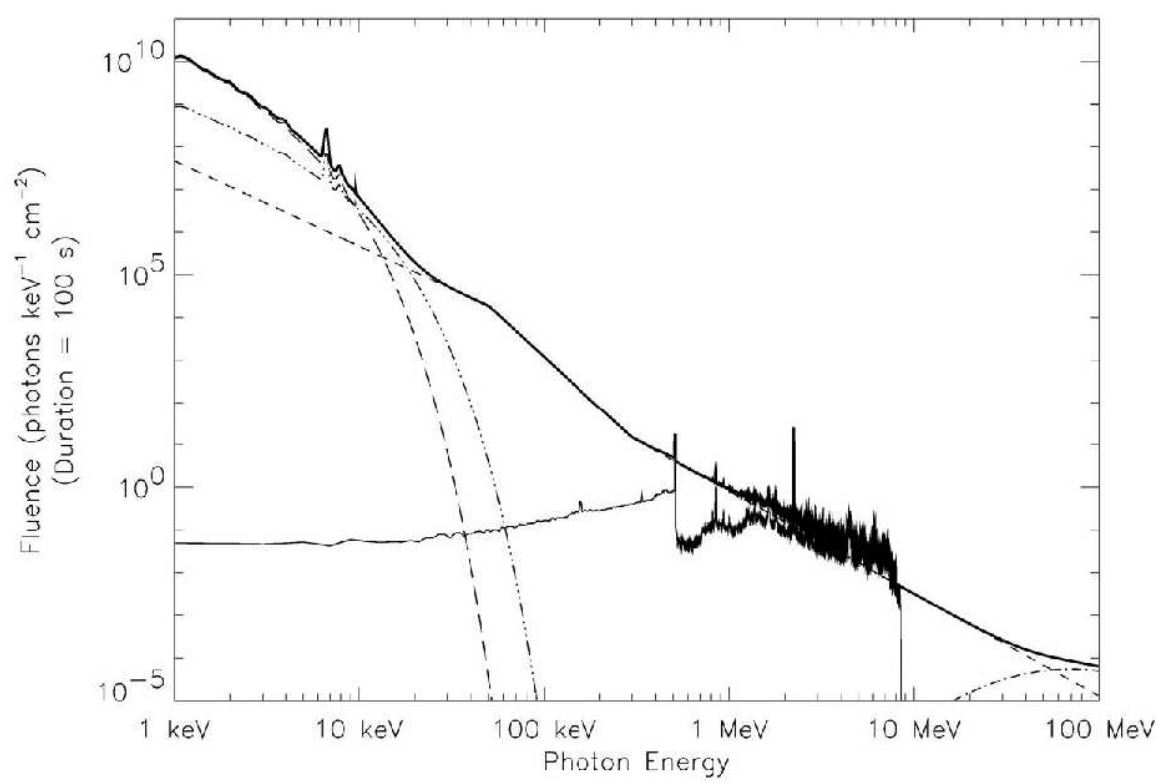

Fig. 6. Composite X-ray/gamma-ray spectrum from $1 \mathrm{keV}$ to $100 \mathrm{MeV}$ for a large flare. At energies up to $10-30 \mathrm{keV}$, emission from hot $\left(10^{7}\right)$ and 'superhot' $\left(3 \times 10^{7}\right)$ thermal flare plasmas (the two curves at the left) dominate. Bremsstrahlung emission from energetic electrons produces the X-ray/gamma-ray continuum (straight lines) up to tens of $\mathrm{MeV}$. Broad and narrow gamma-ray lines from nuclear interactions of energetic ions sometimes dominate the spectrum between 1 to $7 \mathrm{MeV}$. Above a few tens of $\mathrm{MeV}$ the photons produced by the decay of pions (curve at the right) dominates. RHESSI observations cover almost four orders of magnitude in energy (3 $\mathrm{keV}$ to $17 \mathrm{MeV}$ ) [104]. 


\section{De-excitation lines}

Flare-accelerated ions (protons, alphas, heavier ions) are responsible for the production of gamma ray emission when they collide with ambient ions and produce excited nuclei that emit nuclear de-excitation lines. Again, the emission process depends on the density of the ambient plasma and therefore the emission is expected from dense regions (i.e. the chromosphere). Since the de-excitation is happening almost instantaneously after the collision, these gamma ray lines are also referred to as prompt lines. Depending on the ratio of the mass of the accelerated ion to the target ion, the line emission can be narrow or broad. Narrow lines are produced when a flare-accelerated proton or alpha particle hit a heavy ambient ion. The width of the emitted line is then produced by the recoil of the heavy ambient ion, and a narrow line is produced. On the other hand, if a heavy flare-accelerated ion hits an ambient proton or alpha particle, the emitted radiation is Doppler-shifted and therefore broad.

RHESSI provides for the first time spectrally resolved observations of narrow de-excitation lines. Statistics are generally limiting the observations, but the narrow lines can still be fitted and the red shift of the lines can be measured. Heavier nuclei are expect to recoil less and therefore show less redshift [155].

\section{Neutron Capture Line}

The most prominent line emission in the gamma ray spectrum is the neutron capture line at $2.223 \mathrm{MeV}$. This line is produced by the capture of thermalized neutrons that were produced by nuclear reactions after flare-accelerated ions hit ambient ions (the dominant neutron production at high energies comes from the breakup of $\mathrm{He}$ ). The thermalized neutrons are captured by ambient protons and a deuterium and a photon at $2.223 \mathrm{MeV}$ are produced. Since the neutrons are thermalized (i.e. have a low velocity) the $2.223 \mathrm{MeV}$ line is very narrow. Since initially the neutrons have to first thermalize before they can be captured, the time profile of the $2.223 \mathrm{MeV}$ line is delayed relative to the prompt lines.

\section{Energy estimates}

The different gamma-ray lines can be used to get information about the flare accelerated ion spectrum. Estimates of the total energy in non-thermal ions can again be derived by integrating over the ion spectrum. The lower energy cutoff, however, is even more uncertain than for the electron spectrum.

\section{Comparing electron and ions acceleration}

Comparing the fluence of $>300 \mathrm{keV}$ emission with the fluence of the $2.2 \mathrm{MeV}$ line, the electron and ion acceleration in flares can be compared. A rough cor- 
RHESSI Spectrum, X4.8 Flore of $7 / 23 / 02,00: 26: 08-00 ; 47 \div 00$

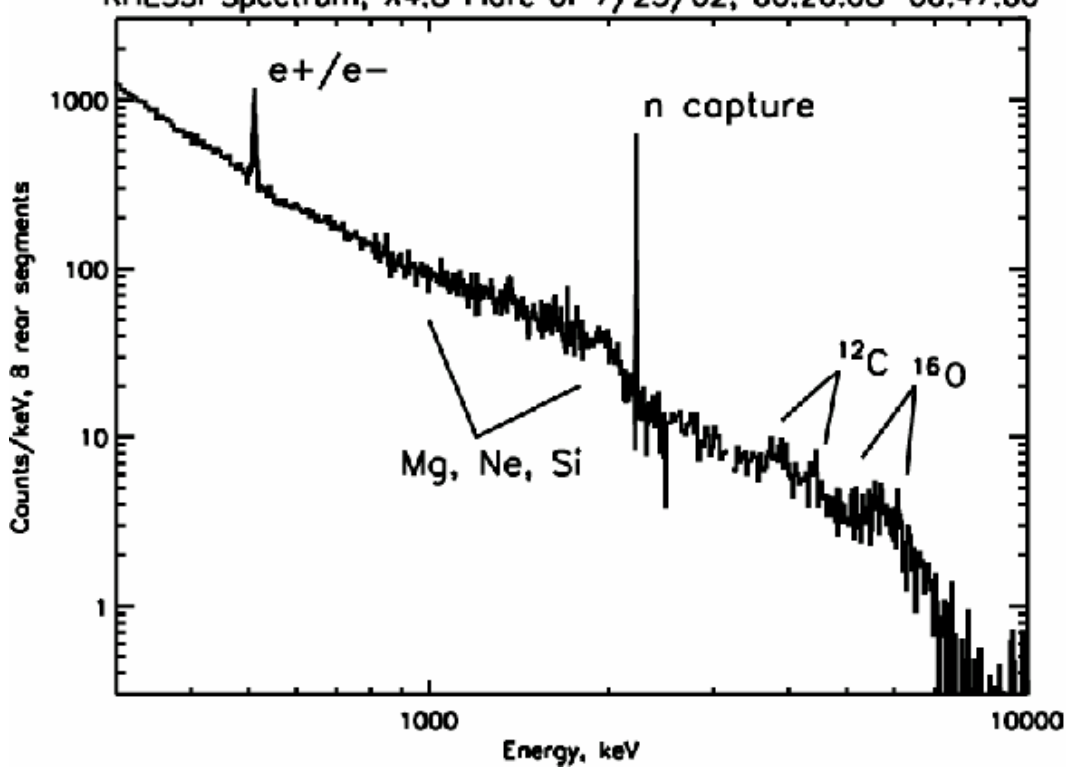

Fig. 7. RHESSI gamma ray spectrum of the July 23, 2002 flare.

relation is observed indicating that at least in very large flares (gamma-ray emission from small flares are not detectable with present day instrumentation), both electrons and ions are always accelerated.

\section{Gamma ray imaging}

RHESSI provides for the first time spatial information of gamma ray emission, the only direct indication of the spatial properties of accelerated ions near the Sun. The most powerful tool for gamma ray imaging is the $2.223 \mathrm{MeV}$ neutron capture line, because of good statistics and a narrow line width which limits the non-solar background to a minimum compared to broad lines. However, the spatial resolution of 35 " is much poorer than the 2" resolution in the hard $\mathrm{X}$-ray range

For the event with best statistics (October 28, 2003 [79]), the $2.223 \mathrm{MeV}$ source structure shows two footpoints similar to the HXR source structure but clearly displaced by $\sim 15 "$ (see Fig. 8 ). This indicates that electrons and ions are accelerated in similar-sized magnetic structures. The displacement could be explained by different accelerator sites for electrons and ions, or by different transport effects from a possibly common acceleration site to the location where the electrons and ions lose their energy by collisions. 


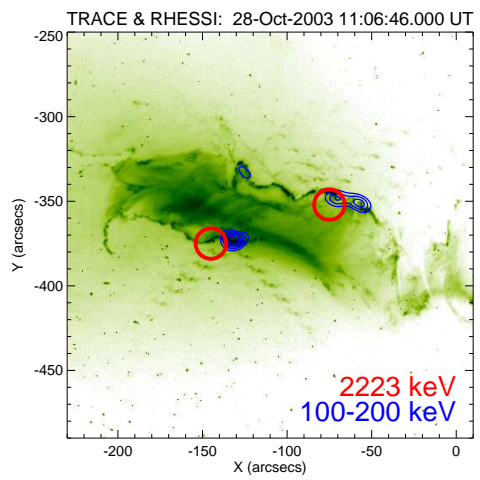

Fig. 8. Imaging of the $2.223 \mathrm{MeV}$ neutron-capture line and the HXR electron bremsstrahlung of the flare on October 28, 2003. The red or gray circles show the locations of the event-averaged centroid positions of the $2.223 \mathrm{MeV}$ emission with $1 \sigma$ uncertainties; the blue or black lines are the 30,50 , and $90 \%$ contours of the $100-200 \mathrm{keV}$ electron bremsstrahlung sources at around 11:06:46UT. The underlying EUV image is from TRACE at $195 \AA$ with offset corrections applied. The gamma-ray and HXR sources are all located on the EUV flare ribbons seen with TRACE.

\subsection{Energetic particles escaping from the sun}

\section{Flare accelerated electrons escaping the Sun}

$\mathrm{X}$-rays are remote sensing diagnostics of energetic electrons that lose their energy by collisions. Upward moving energetic electrons that have access to field lines extending into interplanetary space (often referred to as "open field lines") only suffer a few collisions (the density is decreasing rapidly) and can therefore escape from the Sun and be observed in-situ near the Earth with particle detectors. These events show fast rise times with slow decays and are called 'impulsive electrons events' when observed near Earth [105]. They are seen with energies from above $1 \mathrm{keV}$ up to the highly relativistic regime. Quite often the first electrons to arrive are observed to travel without suffering any collisions (ballistic transport) and they are therefore referred to as "scatterfree" events. The ballistic transport means that high energy electrons arrive earlier than lower energy ones, indicating that electrons at all energies left the Sun around the same time. The observed dispersion in the onset times of 
the different energy channels can therefore be used to approximate when the energetic electrons left the Sun. In some case (about one third of all events) a clear temporal correlation exists with the occurrence of HXR emission during solar flares and the release of energetic electrons into interplanetary space (Fig. 9a).

This indicates that possibly the same acceleration mechanism produces the energetic electrons that create HXR emission in the chromosphere and those energetic electrons that escape into interplanetary space. This picture can be further corroborated by comparing the HXR spectrum with the in situ electron spectrum. If the chromospheric X-ray spectrum is flat (hard), the electron spectrum observed near Earth ia also flat (Fig. 9b).
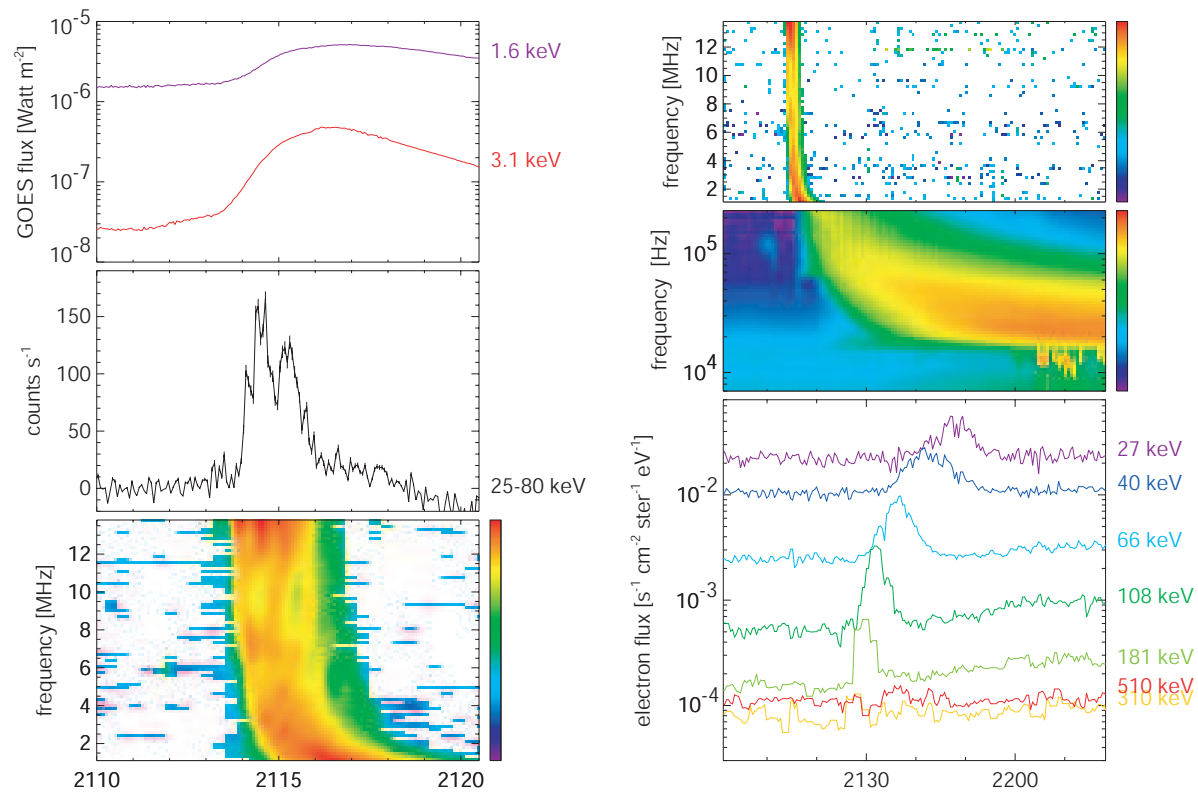

Fig. 9. Impulsive electron event observed on October 19, 2002: (left) From top to bottom, GOES soft X-ray light curves, RHESSI 25-80 keV light curve, and WIND/WAVES radio spectrogram in the 1 to $14 \mathrm{MHz}$ range are shown [29]. (right) An expanded view of the WIND/WAVES data including low frequency observations is presented in the top two panels, while the bottom panel shows in-situ observed energetic electrons from 30 to $500 \mathrm{keV}$ detected by WIND/3DP. This event (like all events selected in this survey) shows a close temporal correlation between nonthermal HXR emission, radio type III emission in interplanetary space, and in-situ observed electrons.

For particles to escape into interplanetary space, they must have access to open field lines. How that happens is not well understood. In the "classic" flare scenario (e.g. [153]) no open field lines are shown. For flares with a 
good temporal and spectral correlation with electron events observed in situ, the flare geometry indeed looks different. These events show hot flare loops with HXR footpoints, plus an additional HXR source separated from the loop by 15 " with only little heating. This source structure can be explained by a simple magnetic reconnection model with newly emerging flux tubes that reconnect with previously open field lines, so-called interchange reconnection. The previously open field lines form the flare loops, while the newly opened field lines show less heating since material can be easily lost because the field is open. Upwards moving energetic electrons escape along the newly opened field line (see Fig. 10).

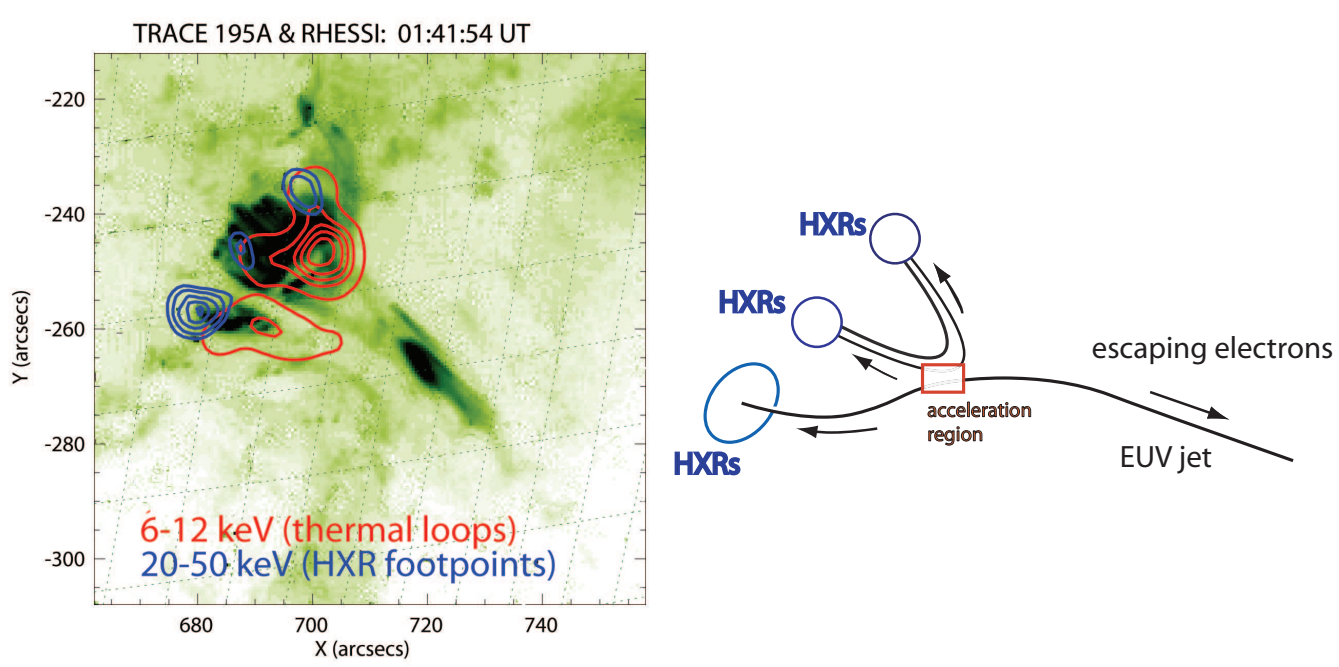

Fig. 10. EUV and X-ray sources of a flare that released energetic electrons into interplanetary space that were later observed near the Earth.

Left figure: RHESSI contours at $6-12 \mathrm{keV}$ (red or dark gray: thermal emission) and $20-50 \mathrm{keV}$ (blue or black: non-thermal emission) overlaid on a TRACE $195 \AA$ EUV image (dark region corresponds to enhanced emission). Located at around $[700,-245]$ arcsec, the X-ray emission outlines a loop with two presumably nonthermal footpoints. The strongest footpoint source however, is slightly to the southeast $[683,-257]$ and shows a surprisingly lower intensity thermal source.

Right figure: Suggested magnetic field configuration showing magnetic reconnection between open and closed field lines inside the red or dark gray box marked as the "acceleration region" where downward moving electrons produce the HXR sources and upward moving electrons escape into interplanetary space. 


\section{Flare accelerated ions escaping from the Sun}

Temporal and spectral comparisons can also be made for ions escaping from the Sun in a similar way to escaping electrons. However, this is much more difficult to do because of the poorer count statistics in the gamma ray range.

The timing of escaping ions is sometimes delayed relative to the flare emission, often significantly (1hour) [94]. Generally it is thought that the shocks of Coronal Mass Ejections are mainly responsible for the energetic ions seen near to the Earth. If this is indeed the case, then a spectral comparison between in-situ observed ion spectra and gamma ray line observations should give no correlation. Surprisingly, in the two gamma-ray line flares observed by RHESSI that are magnetically well-connected (2 Nov 2003 and 20 Jan 2005), the spectrum of the energetic protons producing the gamma-ray lines was found to be essentially the same as that of the SEP protons observed at $1 \mathrm{AU}$. These two events had quite different spectral slopes, so this agreement is unlikely to be a coincidence. It suggests that the gamma-ray producing and in-situ energetic protons may have the same source (at least in these two events), contrary to the standard two-class paradigm (i.e. flare accelerated and CME accelerated ions). These results illustrate the present lack of physical understanding regarding the SEP acceleration process(es).

\subsection{Statistical properties of flares}

Flares are not just simple explosions in the solar atmosphere. Even a single "flare" shows many individual peaks during its evolution [70, 71]. When observing an active region or the whole Sun for a certain period of time, a number of flares with different total energy $\mathrm{E}$ (or peak energy $E_{p}$ ) will be recorded. If we define as $F(E) d E$ the fraction of flares which released energy between $E$ and $E+d E$, then a very striking statistical feature of energy release in active regions emerges [38]. The frequency distribution $F(E)$ reconstructed from UV, EUV and X-ray observations has a simple form (see Fig. 11)

$$
F(E)=F_{0} E^{-a}
$$

which holds for eight orders of magnitude in E. Similar laws are obtained for the peak energy and the flare duration. The value of the exponent is not constant and may range from $1.6-2.0$, depending of the data set used. Current instruments are not able to observe nano-flares (energies below $10^{24}$ ergs) and the lower part of the distribution, which plays a crucial role in coronal heating, is uncertain. A key point for our discussion here is that the energy release of the active region is self similar. This particular feature of the observed characteristics of flares has created many heated discussions and remain an open and difficult theoretical problem which will be discussed in the next section. 

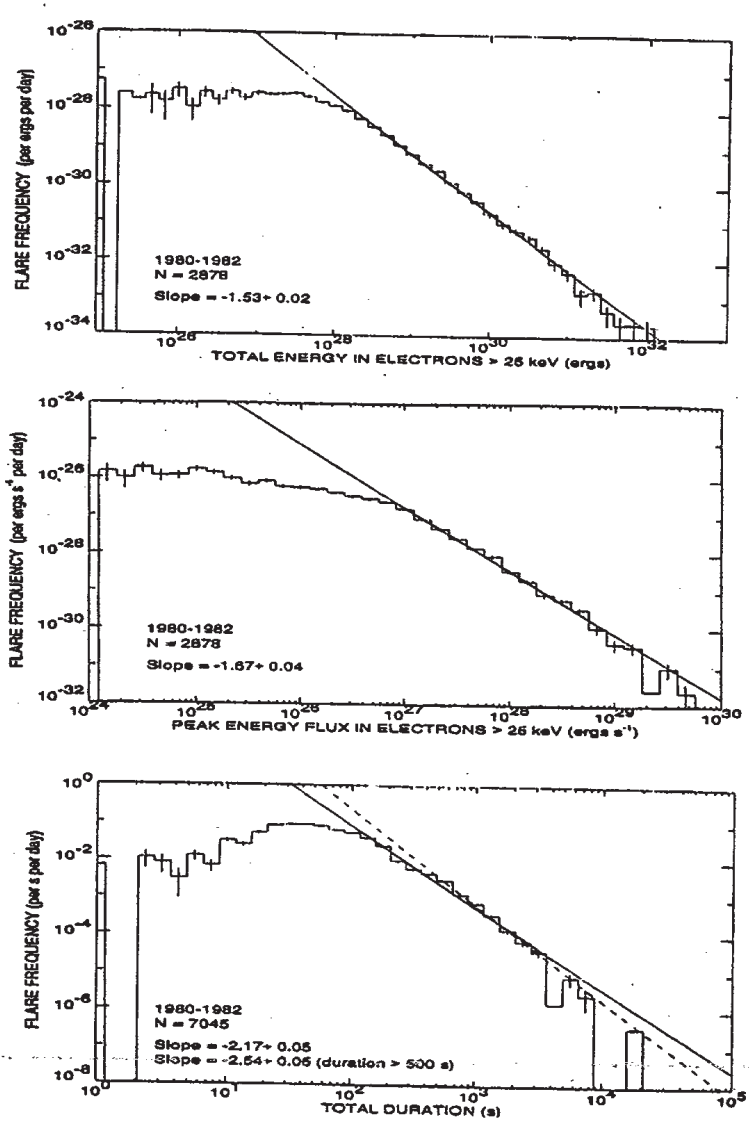

Fig. 11. The frequency distribution of total flare energy, peak flux and duration $[38]$.

\subsection{Summary of observational constrains and discussion}

We now pull together the above results, and address their implications for understanding flare particle acceleration. Of particular importance are the implications arising from the thick target model:

1. The thick target model for HXR and its theoretical implications: The theoretical basis of the thick target model, as originally presented [30] and re-iterated recently [31], is based on the assumption that the accelerator is located in the corona and the HXR source in the upper chromosphere. Thus the acceleration region is collision-free and the radiation source is collision-dominated and electrons travel the distance between acceleration region and radiation region ballistically [16]. A large HXR burst flux suggests that the required electron flow rate is $\geq 10^{37}$ 
electrons/sec with electron energies above $20 \mathrm{keV}$. This amounts to a total of $10^{39}$ electrons for a burst lasting several minutes. This result, known as the number problem, implies that all the particles inside a very large coronal volume $\left(\sim 10^{30} \mathrm{~cm}^{3}\right.$, almost the entire corona above an active region) are accelerated within a few minutes and stream towards the chromosphere. Assuming that the acceleration is inside a large-scale current sheet (see Fig. 12 and later discussion) with typical dimensions $10^{10} \mathrm{~cm} \times 10^{10} \mathrm{~cm} \times 10^{5} \mathrm{~cm}$, this monolithic current sheet must accelerate all the particles entering it (the inflow velocity needs to be a fraction of the local Alfven speed) and remain stable for tens of minutes. We return to these points at the end of the Section.

2. Energetics: Assuming that $\sim 10^{39}$ electrons are accelerated with a mean energy of $50 \mathrm{keV}$, the energy they carry is $\sim 10^{31}$ ergs. Since the accelerated particle fill a volume $\sim 10^{30} \mathrm{~cm}^{3}$ and if the mean magnetic field available for dissipation in the corona is $30 \mathrm{G}$, the available magnetic energy is $\sim 5 \times 10^{31}$ ergs so a significant fraction of the magnetic energy in this acceleration volume will go to the energetic electrons

3. Spectral index and low energy cut-off: The energetic particles form a thermal distribution up to a critical energy $E_{c} \sim 1-30 \mathrm{keV}$ and a power law distribution above this energy. The spectral index $(\delta)$ varies both in the course of the burst and from event to event but remains within the range 2-6. The presence of multiple breaks at different energies is also observed frequently.

4. The temporal evolution of the power law index: The power law index varies during the impulsive phase of the flare, following a specific evolution: soft-hard-soft.

5. Acceleration time: The accelerator should start on sub-second timescales and remain on for tenths of minutes for the electrons. Ions are also accelerated in secs and the accelerator remains active (sometimes) for hours.

6. Maximum energy: The maximum energy achieved is close to hundreds of $\mathrm{MeV}$ for the electrons and several $\mathrm{GeV}$ for the ions.

7. Flare statistics: The flares released in a specific active region are not random. They follow a specific statistical law in energy, peak intensity and duration.

8. Footpoint motion: According to the "standard model" (see below) reconnection causes the footpoints to move smoothly away from each other or along the filament. Some observations seem to support this prediction but others not, so the motion of the footpoints is still an open question.

9. The coronal sources: Coronal sources at $20-30 \mathrm{keV}$ are hard to confine collisionally, therefore the fact that they persist as isolated blobs in space, their characteristic spectral evolution, and their movement, remain open theoretical challenges.

10. The close time and spectral evolution of the two footpoints: When the two footpoints appear (usually in energies above $30 \mathrm{keV}$ ), they seem to correlate in temporal and spectral evolution leaving the impression 
that the accelerated particles moving in them are coming from the same acceleration source.

11. Interplanetary energetic particles: There is a close correlation of the HXR index with the properties of energetic particles detected in the interplanetary medium. This appears to need more complex magnetic topologies that currently discussed at the Sun.

12. High energy Ions: There is an observed shift in the location of ion and electron footpoints. Sometimes, contrary to the electrons, the energetic ions show a single foot point. The acceleration of ions and electrons in different length loops and the loop anisotropy with the low sensitivity are two explanations offered so far. There is an apparent correlation between electron acceleration above $300 \mathrm{keV}$ and ion acceleration. The correlation of relativistic electron and ions, and the fact that the spectrum of electrons above $300 \mathrm{keV}$ remains a power law with harder spectrum, recalls an older suggestion for two-stage acceleration, where shock acceleration may play an active role in the second stage in some large flares.

From the above summary, several important points arise, many concerning the efficiency requirements of the thick target model. It is especially interesting to discuss this in the context of what is sometimes referred to the "standard flare mode" as shown in Fig. 12. This orignated in old models for long-decay flares $[33,90]$, and has been proposed as a generic scenario for coronal flaring. In particular, the model invokes a monolithic current sheet, which, one must assume, is where the particle acceleration takes place. In fact, as we will show in the next section, it is rather difficult to achieve efficient acceleration in simple magnetic topologies.

There are major electrodynamic constraints arising in the thick target model. The large flux of energetic electrons $\left(F_{37} \sim 10^{37}\right.$ electrons $\left./ \mathrm{sec}\right)$ flowing through a relatively small area (the observed footpoints are relatively compact with characteristic area $\left.A_{17} \sim 10^{17} \mathrm{~cm}^{2}\right)$ suggests that the beam density of the energetic electrons (mean velocity $10^{10} \mathrm{~cm} / \mathrm{sec}$ ), can be as high as $n_{b} \sim$ $10^{10}$ electrons $/ \mathrm{cm}^{3}$. Assuming that the ambient density at the HXR source is comparable or one order of magnitudes higher $\left(n_{0} \sim 10^{11}\right.$ particles $\left./ \mathrm{cm}^{3}\right)$, a neutralising return current is required with a characteristic velocity of $v_{r} \sim$ $10^{9} \mathrm{~cm} / \mathrm{sec}$. The return current replenishes the already-accelerated particles in the acceleration volume with hot plasma if the acceleration region and radiation region are magnetically connected. The observed hot thermal loops and the Neupent effect can be the observational tests for the reaction of the chromosphere to this intense electron beam injected from the corona. This vital point is not incorporated in current flare models, and the problem of particle replenishment remains an open issue.

We also note that the scenario adopted for the thick target model for HXR and the "standard flare model" leave number of open questions: (1) How is a correlation between HXR and Type III bursts established? (2) The density of the beams driving the normal type III burst $\left(n_{b}(I I I) \sim 10^{6}\right.$ electrons $\left./ \mathrm{cm}^{3}\right)$ are 


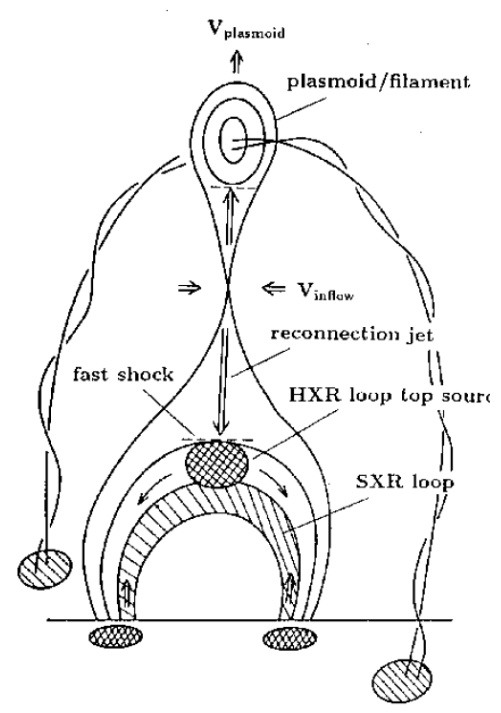

Fig. 12. This cartoon, suggested several years ago remains the favorite model and was elevated recently to the "standard flare cartoon". It has been in the literature for many years, it was revised to incorporate more recent observation and it has been born out in simple 2-D simulations. [153]

several orders of magnitude less than the beam density needed to power the HXR through the thick target $\left(n_{b}(H X R) \sim 10^{10}\right.$ electron $\left./ \mathrm{cm}^{3}\right)$. What caused this large imbalance? (3) The chromospheric evaporation will refill the loops with plasma in seconds, but if the acceleration and the energy release is above the loop(s) and the collapsing process for the formation of the loops has been completed, how is the plasma inside the loop is re-accelerated?

We conclude that the standard 2-D flare cartoon shown in Fig. 12 and/or 2-D simulations based on the cartoon, are not able to handle the relevant physics question. Eruption in 3-D magnetic topologies is still an active research project and the simple magnetic topology presented in Fig. 12 can mainly be used to represent an idea of how the overall magnetic structure may respond when energy is released during a CME/flare. We will return on this issue in the next section.

The above constraints for the energy release and the subsequent acceleration of high energy particles during large flares are hard to reconcile with reconnection theory as hosted in a simple magnetic topology and associated with a particular acceleration mechanism (DC electric fields, shocks or MHD waves). However, this discussion should not be construed as an objection to the role of reconnecting current sheets in flare acceleration and particle trapping per se. It is a characteristic of the magnetohydrodynamic equations that 
they are "self-similar" over a wide range of scales: in other words the acceleration and heating is not just restricted to the large monolithic sheet, but occurs at current sheets of all sizes. In the following sections we discuss in more detail recent developments in the formation of the magnetic environment for particle acceleration, and try to relate these topologies with mechanisms for particle acceleration.

\section{Models for impulsive energy release}

\subsection{3-D extrapolation of magnetic field lines and the formation of unstable current sheets}

The energy needed to power solar flares is provided by photospheric and subphotospheric motions and is stored in non-potential coronal magnetic fields. Since the magnetic Reynolds number is very large in the solar corona, MHD theory states that magnetic energy can only be released in localized regions where the magnetic field forms small scales and steep gradients, i.e. in thin current sheets (TCS).

Numerous articles (see recent reviews $[46,107]$ ) are devoted to the analysis of magnetic topologies which can host TCSs. The main trend of current research in this area is to find ways to realistically reconstruct the 3-D magnetic field topology in the corona based on the available magnetograms and large-scale plasma motions at the photosphere. One must then search for the location of special magnetic topologies, i.e. separatrix surfaces (places were field lines form null points [99] and bald patches [158]), and more generally Quasi-Separatrix Layers (QSL) which are regions with drastic changes of the field line linkage [46]. A variety of specific 3-D magnetic configurations (fans, skeletons etc) have been analyzed, and their ability to host fast diffusion of the magnetic field lines has also been investigated [143]. The main analytical and computational approaches through which these structures are analysed are based on prescribed and simple magnetic structures at the photosphere, e.g. a quadrupole [17] (see Fig. 13). A realistic magnetic field generates many "poles and sources" [107] and naturally has a relatively large number of TCSs. We feel that this detailed representation of topological forms of the TCSs is mathematically appealing for relative simple magnetic topologies (dipoles, quadrupoles, symmetric magnetic arcades [17]). When such topological simplicity at the photosphere is broken, for example due to large-scale sub-Alfv́enic photospheric motions or the emergence of new magnetic flux that disturbs the corona, such tools may be less useful. All these constraints restrict our ability to reconstruct fully the dynamically evolving magnetic field of an active region (and it is not clear that such a reconstruction will ever be possible).

Many of the widely used magnetograms measure only the line of sight component of the magnetic field. The component of the magnetic field vertical 


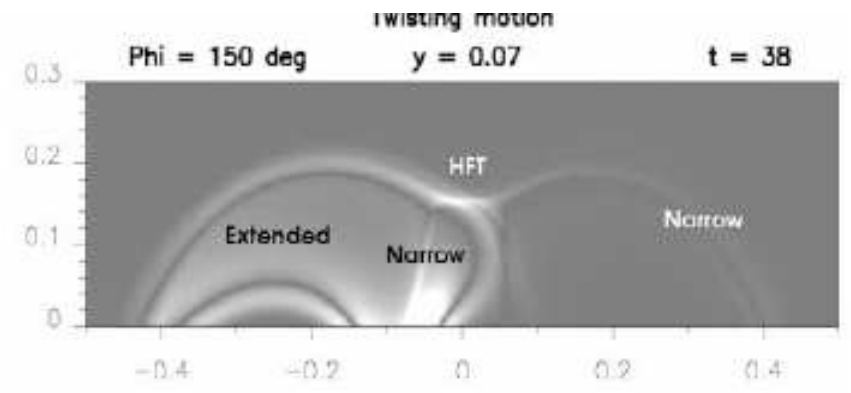

Fig. 13. Projected view of the two stressed magnetic field configurations used as initial conditions for the search of QSL's [17] .

to the surface matches the measured magnetic field only at the center of the disk and becomes increasingly questionable as the limb is approached. Extrapolating the measured magnetic field is relatively simple if we assume that the magnetic field is a force-free equilibrium:

$$
\nabla \times \boldsymbol{B}=\alpha(\boldsymbol{x}) \boldsymbol{B}
$$

where the function $\alpha(\boldsymbol{x})$ is arbitrary except for the requirement $B \cdot \nabla \alpha(\boldsymbol{x})=0$, in order to preserve $\nabla \cdot \boldsymbol{B}=0$. Eq. (2) is non-linear since both $\alpha(\boldsymbol{x})$ and $\boldsymbol{B}(\boldsymbol{x})$ are unknown. We can simplify the analysis of Eq. 2 when $\alpha=$ constant. The solution is easier still when $\alpha=0$, which is equivalent to assuming the coronal fields contain no currents (potential field), hence no free energy, and thus uninteresting.

A variety of techniques have been developed for the reconstruction of the magnetic field lines above the photosphere and the search for TCSs [107, 113]. It is beyond the scope of this article to discuss these techniques in detail. For instructive purposes, we use the simplest method available, a linear force free extrapolation, and search for "sharp" magnetic discontinuities in the extrapolated magnetic fields. Vlahos and Georgoulis [171] use an observed active-region vector magnetogram and then: (i) resolve the intrinsic azimuthal ambiguity of $180^{\circ}$ [65], and (ii) find the best-fit value $\alpha_{A R}$ of the force-free parameter for the entire active region, by minimizing the difference between the extrapolated and the ambiguity-resolved observed horizontal field (the "minimum residual" method of [102]). They perform a linear force-free extrapolation [6] to determine the three-dimensional magnetic field in the active region. Although it is known that magnetic fields at the photosphere are not force-free [67], they argue that a linear force-free approximation is suitable for the statistical purposes of their study.

Two different selection criteria were used in order to identify potentially unstable locations (identified as the afore-mentioned TCSs) [171]. These are (i) the Parker angle, and (ii) the total magnetic field gradient. The angular difference $\Delta \psi$ between two adjacent magnetic field vectors, $\mathbf{B}_{\mathbf{1}}$ and $\mathbf{B}_{\mathbf{2}}$, is 
given by $\Delta \psi=\cos ^{-1}\left[\mathbf{B}_{\mathbf{1}} \cdot \mathbf{B}_{\mathbf{2}} /\left(B_{1} B_{2}\right)\right]$. Assuming a cubic grid, they estimated six different angles at any given location, one for each closest neighbors. The location is considered potentially unstable if at least one $\Delta \psi_{i}>\Delta \psi_{c}$, where $i \equiv\{1,6\}$ and $\Delta \psi_{c}=14^{\circ}$. The critical value $\Delta \psi_{c}$ is the Parker angle which, if exceeded locally, favors tangential discontinuity formation and the triggering of fast reconnection $[136,137]$. In addition, the total magnetic field gradient between two adjacent locations with magnetic field strengths $B_{1}$ and $B_{2}$ is given by $\left|B_{1}-B_{2}\right| / B_{1}$. Six different gradients were calculated at any given location. If at least one $G_{i}>G_{c}$, where $i \equiv\{1,6\}$ and $G_{c}=0.2$ (an arbitrary choice), then the location is considered potentially unstable. When a TCS obeys one of the criteria listed above, it will be transformed to an Unstable Current Sheet (UCS). A steep gradient of the magnetic field strength, or a large shear, favors magnetic energy release in three dimensions in the absence of null points [144]. [Note that these thin elongated current sheets have been given different names by different authors: e.g. in [17] they are called Hyberbolic Flux Tubes.]
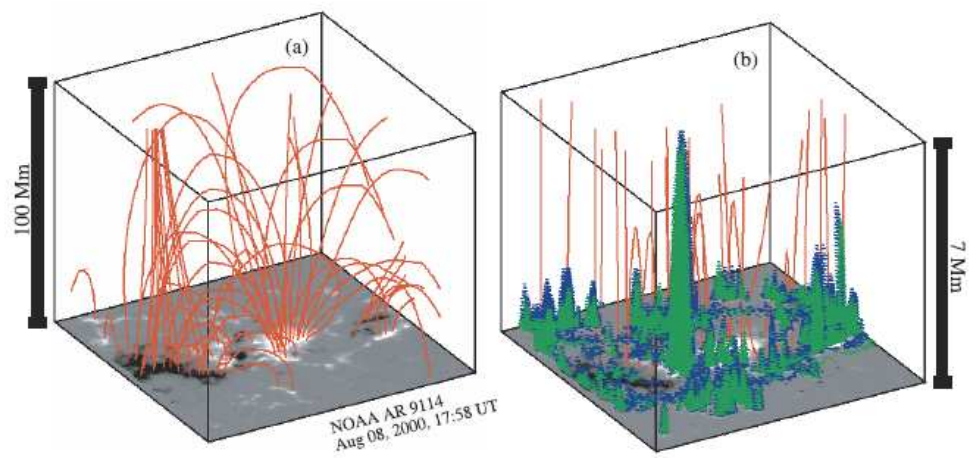

Fig. 14. (a) Linear force free field extrapolation in NOAA AR 9114, (b) Lower part of the AR atmosphere. Shown are the magnetic field lines (red) with the identified discontinuities for critical angle $10^{\circ}[171]$.

Potentially unstable volumes are formed by the merging of adjacent selected locations of dissipation. These volumes are given by $V=N \lambda^{2} \delta h$, where $N$ is the number of adjacent locations, $\lambda$ is the pixel size of the magnetogram and $\delta h$ is the height step of the force-free extrapolation. The free magnetic energy $E$ in any volume $V$ is given by

$$
E=\frac{\lambda^{2} \delta h}{2 \mu_{0}} \sum_{l=1}^{N}\left(\mathbf{B}_{\mathrm{ff} l}-\mathbf{B}_{\mathbf{p}_{l}}\right)^{2}
$$

where $\mathbf{B}_{\mathbf{f f} l}$ and $\mathbf{B}_{\mathbf{p}_{l}}$ are the linear force-free and the potential fields at location $l$ respectively. The assumption used is that any deviation from a potential 
configuration implies a non-zero free magnetic energy which is likely to be released if certain conditions are met. UCS are created naturally in active

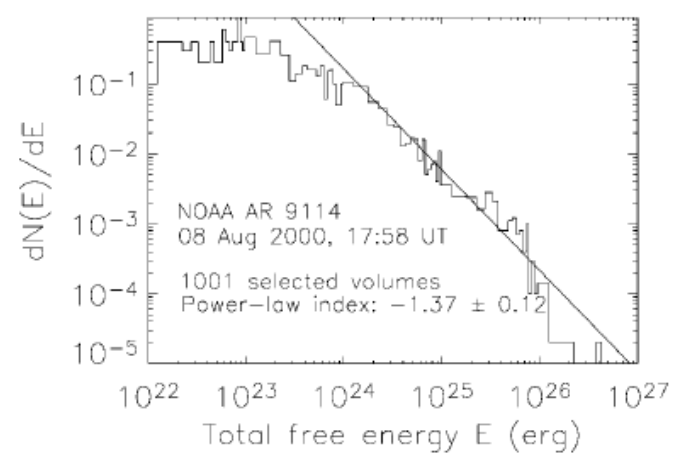

Fig. 15. Typical distribution function of the total free energy in the selected volume, on using a critical angle $14^{\circ}[171]$.

regions even during their formation (Fig. 14) and the free energy available in these unstable volumes follows a power law distribution with a well defined exponent (Fig. 15). We can then conclude that active regions store energy in many unstable locations, forming UCS of all sizes (i.e. the UCS have a selfsimilar behavior). The UCS are fragmented and distributed inside the global 3 -D structure. Viewing the flare in the context of the UCS scenario presented above, we can expect, depending of the size and the scales of the UCS, to have flares of all sizes. Small flares dominate, and have the potential to heat the corona, and large flares occur when large-scale QSL complexes are formed.

The next step is to analyze the evolution of an isolated UCS. We already stressed above that the method followed by [171] has several weak points, but nevertheless provides a simple tool for the analysis of the statistical behaviour of the places hosting UCS and flares (see also [108]). Aulanier et al $[17,18]$ started from a carefully prepared magnetic topology in the photosphere (bipolar formed by four flux concentration regions) in which the potential extrapolation contains QSLs, and observed and analyzed the formation and the properties TCSs. The 3-D magnetic topology was driven by photospheric motions and the end result was the formation of TCSs in the vicinity of the QSLs. Unfortunately no statistical analysis of the characteristics of the TCSs were reported since the MHD codes used do not have the ability to resolve the transition from TCSs to UCSs.

\subsection{The 3-D turbulent current sheet}

Magnetic reconnection is the topological change of a magnetic field by the breaking the magnetic field lines. It happens in regions where the assump- 
tion of flux freezing in ideal magnetohydrodynamics (MHD) no longer holds $[142,26]$. Resistivity plays a key role in magnetic reconnection. The classical (Spitzer) resistivity in the solar corona is extremely low $\left(\sim 10^{-16}\right)$ therefore ideal MHD theory holds in general. Exceptions are the UCS where the resistivity can jump by many orders of magnitude and ideal MHD theory becomes invalid $[132,46]$. [Of course the UCS should be analyzed ideally in the framework of 3-D kinetic theory [28, 177].]

Onofri et al. [132] studied the nonlinear evolution of current sheets using the 3-D incompressible and dissipative MHD equations in a slab geometry. The resistive MHD equations in dimensionless form are:

$$
\begin{aligned}
\frac{\partial \boldsymbol{V}}{\partial t}+(\boldsymbol{V} \cdot \nabla) \boldsymbol{V} & =-\nabla\left(P+\frac{B^{2}}{2}\right)+(\boldsymbol{B} \cdot \nabla) \boldsymbol{B}+\frac{1}{R_{v}} \nabla^{2} \boldsymbol{V} \\
\frac{\partial \boldsymbol{B}}{\partial t} & =-\nabla \times \boldsymbol{E} \\
\boldsymbol{j} & =\nabla \times \boldsymbol{B} \\
\boldsymbol{E}+\boldsymbol{V} \times \boldsymbol{B} & =\frac{1}{R_{M}} \boldsymbol{j} \\
\nabla \cdot \boldsymbol{V} & =0 \\
\nabla \cdot \boldsymbol{B} & =0
\end{aligned}
$$

where $\boldsymbol{V}$ and $\boldsymbol{B}$ are the velocity and the magnetic field, respectively, $\mathrm{P}$ is the pressure and $R_{v}$ and $R_{M}$ are the kinetic and magnetic Reynolds numbers with $R_{v}=5000$ and $R_{M}=5000$. Here the density has been set to unity (incompressible) and the constant $\mu_{0}$ absorbed into the magnetic field. The initial conditions were established in such a way as to have a plasma that is at rest in the frame of reference of the computational domain, permeated by an equilibrium magnetic field $\boldsymbol{B}_{\mathbf{0}}$, sheared along the x-direction, with a current sheet in the middle of the simulation domain:

$$
\boldsymbol{B}_{\mathbf{0}}=B_{y o} \hat{y}+B_{z o}(x) \hat{z}
$$

where $B_{y o}$ is constant and $B_{z o}$ is given by

$$
B_{z o}(x)=\tanh \left(\frac{x}{a}\right)-\frac{x / 0.1}{\cosh ^{2}(a / 0.1)}
$$

In the $\mathrm{y}$ and $\mathrm{z}$ directions, the equilibrium magnetic field is uniform and periodic boundary conditions are imposed, since no boundary effects are expected in the development of the turbulence. In the inhomogeneous x-direction, fixed boundary conditions are imposed. These equilibrium fields were perturbed with 3-D magnetic field fluctuations satisfying the solenoidal condition.

The nonlinear evolution of the system is characterized by the formation of small scale structures, especially in the lateral regions of the computational domain, and coalescence of magnetic islands in the center. This behavior is 
reflected in the 3-D structure of the current (see Fig. 16), which shows that the initial equilibrium is destroyed by the formation of current filaments, with a prevalence of small scale features. The final stage of these simulations is a turbulent state, characterised by many spatial scales, with small structures produced by a cascade with wavelengths decreasing with increasing distance from the current sheet. In contrast, inverse energy transfer leads to the coalescence of magnetic islands producing the growth of two-dimensional modes. The energy spectrum approximates a power law with slope close to 2 at the end of the simulation. Similar results have been reported by many authors using several approximations [53,119, 98, 154]. It is also interesting to note that similar results are reported from magnetic fluctuations in the Earth's magnetotail [178].

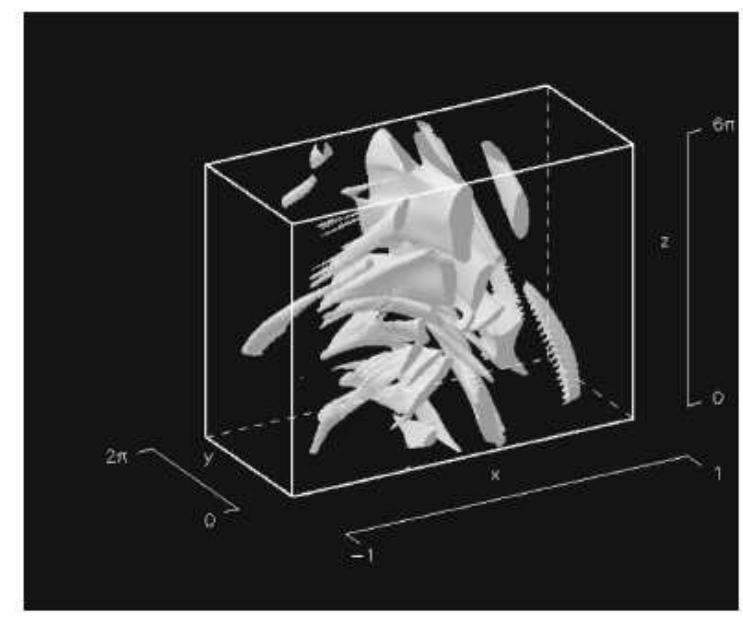

Fig. 16. Current isosurfaces showing the formation of current filaments, [132]

It has become apparent over the years that the (theoretical) Ohm's law used in resistive MHD:

$$
\boldsymbol{E}+\boldsymbol{V} \times \boldsymbol{B}=\eta \boldsymbol{j}
$$

where $\boldsymbol{E}$ and $\boldsymbol{B}$ are the electric and magnetic fields, $\boldsymbol{V}$ is the fluid velocity, $\boldsymbol{j}$ is the current and $\eta$ is the resistivity, breaks down near reconnection sites. The main reason is that the region of electron demagnetization is much smaller than the ion inertial length $c / \omega_{i}$, where c is the speed of light and $\omega_{i}$ the ion plasma frequency, and so Hall terms in the full version Ohm's law become important:

$$
\frac{1}{\omega_{e}^{2}} \frac{d \boldsymbol{j}}{d t}=\boldsymbol{E}+\boldsymbol{V} \times \boldsymbol{B}-\frac{1}{n e} \boldsymbol{j} \times \boldsymbol{B}
$$




$$
+\frac{1}{n e} \nabla \cdot \overrightarrow{P_{e}}-\eta \boldsymbol{j}
$$

where $\overrightarrow{P_{e}}$ is the electron pressure tensor and $\mathrm{n}$ is the plasma density. The proper framework to study magnetic reconnection, including the important contribution of the Hall term in the analysis, is the two-fluid equations. Cessak et al. [36], using a two fluid code, reported a very interesting scenario for magnetic reconnection. The reconnection proceeds slowly and allows the system to accumulate stresses as it forms TCSs which evolve and remain stable over a long period of time. When the thickness of the TCS reaches a critical value, the system adjusts abruptly to exhibit fast reconnection. The authors called this particular model for reconnection "A catastrophe model for fast reconnection", we have adopted here the term UCS for the fast reconnection and the slow evolving mode is called TCS. Switching from the "stable" TCS to fast reconnection (the UCS) is related to the fact that the anomalous resistivity turns on. The need for a critical threshold is thus crucial for the nonlinear evolution of an active region.

Simulating magnetic reconnection with a 3-D full particle code is currently an ongoing research project that presents many difficulties (see [145, 28, 177] and references therein). Experimental verification of magnetic reconnection has shown evidence of a positive correlation between the magnitude of magnetic fluctuations up to the lower-hybrid frequency range [85], and in the Hall effect. They also measure short coherent lengths indicating a strongly nonlinear nature of the evolution of the reconnection current sheet. The main difficulty with a realistic analysis of magnetic reconnection using 3-D kinetic models is the wide range of spatial and temporal scales separating the reconnection region from the magnetic fields observed during a flare or a CME.

\subsection{The compact flare}

A series of recent studies explored the question "How does a loop respond to a random photospheric driver?" In the past, flares were assumed to be driven by organized and continuous twisting or shearing motions in the photosphere. Galsgaard and Nordlund [62] and Galsgaard [63] explored a different scenario for flare initiation. The three-dimensional time-dependent MHD equations (Eqs. 4-9) were solved in a cartesian box with model photospheres at either end. An energy equation with anisotropic heat condition and optical thin radiation is included. Between photosphere and corona there is a stratified atmosphere (the gravitational force is modeled by a sine function, vanishing at the center of the computational box), so that the density profile is a hyperbolic tangent. At the start of each simulation, there is a uniform magnetic field extending between the two photospheric regions. In order to relate the simulations to observed coronal loop structures, the simulation box is 20 times longer than it is wide. The coronal density is $10^{3}$ times smaller than that in the photosphere, implying an Alfvén speed in the corona approximately 30 times larger than in the photosphere. 


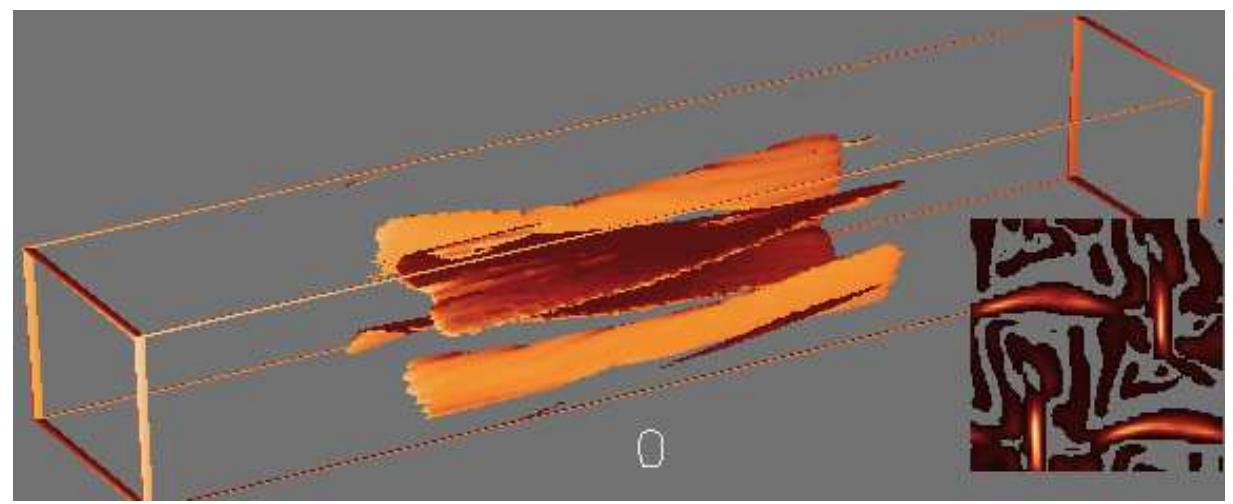

Fig. 17. The loop is stressed by random photospheric flows and is led to a state where numerous current sheets are present. A vertical cross section through the middle of the loop shows the formation of current sheets [63]

Solar magnetic flux tubes connect different regions in the photosphere. This initial state is perturbed by imposing simple sinusoidal shear motions on the magnetic field at the two boundaries. Their wavelength is equal to the transverse length, while their phase, orientation and direction are random. This, in a simple manner, represents the advection of magnetic flux due to convective motions, and injects energy into the corona. The coronal field responds to these boundary motions, with the Lorentz force determining its evolution. After some time (a few seconds corresponding to the time needed for an Alfvén wave to cross the loop) the stresses are distributed along the entire loop, and coronal current sheet (TCS) formation occurs. As reconnection commences (the sudden formation of UCSs), plasma jets are formed, and eventually their momentum is sufficient to strongly perturb the neighboring plasma, creating secondary current concentrations. A turbulent cascade is thus initiated so that throughout the simulation, energy is injected on large scales, but cascades through a turbulent process to the shortest possible length scale where it is dissipated in numerous small current concentrations randomly distributed throughout the volume (see Fig. 17). It was also discovered that the response of the small compact loops (length around $\sim 5 \times 10^{9} \mathrm{~cm}$ ) is to form fragmented current sheets in the middle part of the loop [63]. For longer loops (length larger than $\sim 10^{10} \mathrm{~cm}$ ) the current sheets form at the foot points. This particular observation may have important consequences on the interpretation of several observed characteristics of flares.

\subsection{A Cellular Automata model for the energy release in the solar corona}

Coronal energy release observed at various wavelengths shows impulsive behaviour with events from flares to bright points exhibiting intermittency in 
time and space. Intense X-ray flare emission typically lasts several minutes to tens of minutes, and only a few flares are recorded in an active region that typically lives several days to several weeks. The flaring volume is small compared to the volume of an active region, regardless of the flare size. Intermittency is the dynamical response of a turbulent system when the triggering of the system is the result of a critical threshold for the instability [36]. In a turbulent system one also expect self organization, i.e. the reduction of the numerous physical parameters (degrees of freedom) present in the system to a small number of significant degrees of freedom that regulates the system's response to external forcing [130]. This is the reason for the success of concepts such as Self Organized Criticality (SOC) [19, 20] in explaining the statistical behavior of flares discussed in Section 2.8. Cellular Automata (CA) models typically employ one free parameter (the magnetic field, vector potential, etc) and study its evolution subject to external perturbations. When a critical threshold is exceeded (when the TCS becomes an UCS), parts of the configuration are unstable, and will restructure to re-establish stability. The rearrangement may cause instabilities in adjacent locations, so the relaxation of the system may proceed as an avalanche-type process. In SOC flare models $[109,110,167]$ each elementary relaxation is viewed as a single magnetic reconnection event, so magnetic reconnection is explicitly assumed to occur with respect to a critical threshold.

In solar MHD an UCS disrupts either when its width becomes smaller than a critical value [141], or when the magnetic field vector forms tangential discontinuities exceeding a certain angle [135], or when magnetic field gradients are steep enough to trigger restructuring [144]. We notice that a critical threshold is involved in all cases: the first process points to the turbulent evolution in the magnetic field configuration and the onset of anomalous resistivity, while the latter two imply magnetic discontinuities caused either by the orientation of the magnetic field vector or by changes of the magnetic field strength. Magnetic field gradients and discontinuities imply electric currents via Ampére's law however, so a critical magnetic shear or gradient implies a critical electric current accumulated in the current sheet which in turn leads to the onset of anomalous resistivity [134, 136].

One way of modeling the appearance, disappearance, and spatial organization of UCS inside a large-scale topology is with the use of the Extended Cellular Automaton (X-CA) model [80, 81, 82]. Fig. 18 illustrates some basic features of the X-CA model. The X-CA model has as its core a cellular automaton model of the sand-pile type and is run in the state of Self-Organized Criticality (SOC). It is extended to be fully consistent with MHD: the primary grid variable is the vector-potential, and the magnetic field and the current are calculated by means of interpolation as derivatives of the vector potential in the usual sense of MHD, guaranteeing $\nabla \cdot \boldsymbol{B}=0$ and $\boldsymbol{J}=\left(1 / \mu_{0}\right) \nabla \times \boldsymbol{B}$ everywhere in the simulated 3-D volume. The electric field is defined as $\boldsymbol{E}=\eta \boldsymbol{J}$, with $\eta$ the diffusivity. The latter usually is negligibly small, but if a threshold in the current is locally reached $\left(|\boldsymbol{J}|>J_{c r}\right)$, then current-driven instabilities 


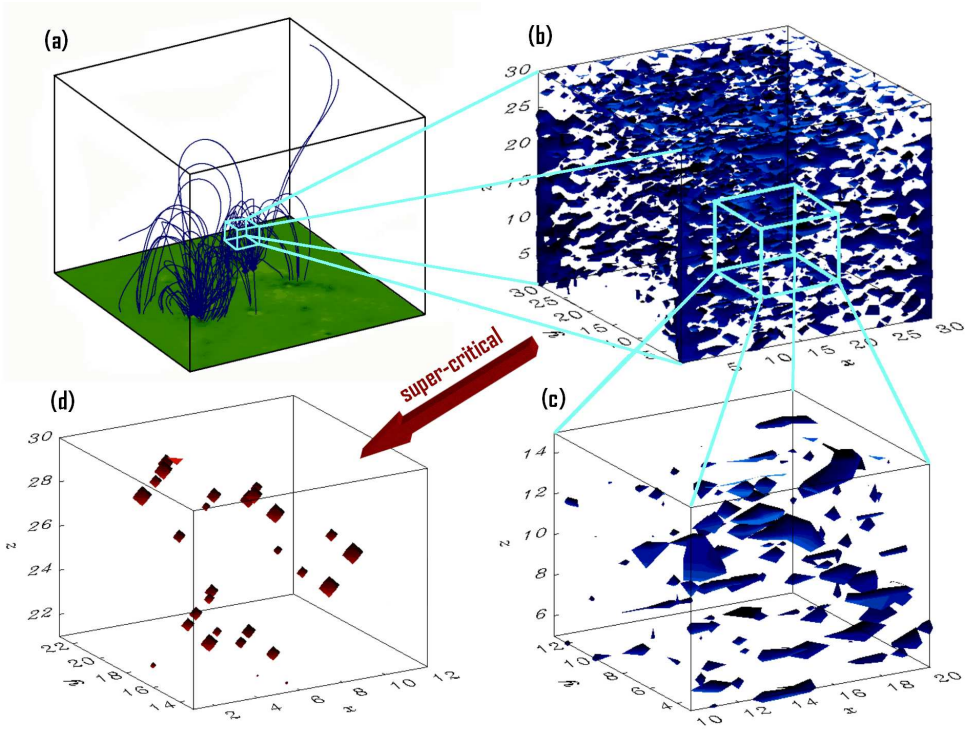

Fig. 18. (a) Simulated magnetogram of a photospheric active region and force-free magnetic field-lines, extrapolated into the corona (b) Sub-critical current iso-surfaces in space, as yielded by the X-CA model, which models a sub-volume of a coronal active region. - (c) The same as (b), but zoomed. (d) Temporal snap-shot of the XCA model during a flare, showing the spatial distribution of the UCS (super-critical current iso-surface) inside the complex active region [172].

are assumed to occur, $\eta$ becomes anomalous in turn, and the resistive electric field locally increases drastically. These localized regions of intense electric fields are the UCS in the X-CA model.

The X-CA model yields distributions of total energy and peak flux which are compatible with the observations. The UCSs in the X-CA form a set which is highly fragmented in space and time: the individual UCS are small scale regions, varying in size, and are short-lived. They do not form in their ensemble a simple large-scale structure, but form a fractal set with fractal dimension roughly $D_{F}=1.8$ [172]. The individual UCS also do not usually split into smaller UCS, but they trigger new UCSs in their neighborhood, so that different chains of UCS travel through the active region, triggering new side-chains of UCS on their way. It is obvious that the rules of this simulation do not include the fragmentation of the UCS and in many ways the results concide with the MHD simulations [63].

\subsection{The magnetic coupling of convection zone with corona}

Active regions are externally driven (from the turbulent convection zone), dissipative (magnetic energy released in coronal heating, flares, CME), nonlinear 
dynamical systems $[169,68]$. Flux emergence and photospheric boundary flows play the role of the driver. The evolution of an active region is largely dictated by the configuration of the magnetic field vector, which is subject to boundary induced perturbations. An important question remains open: is the structure and evolution of magnetograms and the photospheric flows responsible for the activity in active region? In other words, can we predict a flare and/or CME using observations from the photosphere?

A variety of well-established observations have analyzed the characteristics of photospheric magnetograms (see $[181,76]$ and references therein). The most striking properties are:

1. The active region magnetic fields form self similar structures, with the area $(A)$. Probability Distribution Functions (PDF) obeying well-defined power laws $P(A) \sim A^{-1.8}$ and with fractal dimensions ranging approximately between $\sim 1.2-\sim 1.7$ are found (see e.g. [72, 73, 117, 118] and references therein).

2. Numerous studies have revealed the multi-fractal nature of active regions $[100,32,101,1,2]$ and their structure function $[2,3]$.

These magnetogram properties are an important diagnostic for the turbulent convection zone dynamics, and as yet are not reproduced in 3-D MHD simulations.

Recently a percolation model was proposed to simulate the formation and evolution of active regions at the photosphere $[174,151]$. In this model, the evolution of the magnetograms is followed by reducing all the complicated convection zone dynamics into three dimensionless parameters. The emergence and evolution of magnetic flux on the solar surface using a 2-D cellular automaton $(\mathrm{CA})$ is probabilistic and based on the competition between two "fighting" tendencies: stimulated or spontaneous emergence of new magnetic flux, and the disappearance of flux due to diffusion (i.e. dilution below observable limits), together with random motion of the flux tubes on the solar surface. This percolation model explains the observed size distribution of active regions and their fractal characteristics [117, 170]. It was later used for the reconstruction of 3-D active regions using the force free approximation and many of the observational details reported in [171] were reproduced [61]. The connection of photospheric activity with the statistical properties of flares has also been simulated by several authors and the results are promising $[138,77,78,163]$.

We have a long way to go before we establish a good understanding of the connection of the driver (photosphere) with the coronal part of an active region [68]. One point is worth stressing: The details of the magnetogram and the large-scale sub-Alfvénic photospheric flows hold many of the secrets of the activity of the active region. The formation and the statistical properties of TCSs and UCSs are in many ways connected with the properties of the "driver". 


\subsection{The eruptive flare/CME model}

A large number and range of models demonstrate the connection between flares and CMEs [7, 13, 60, 90, 148, 175, 14, 64]. All start from simple (arcade, loop or emerging flux) magnetic topology (analyzed mostly in 2-D and only recently in 3-D) which is driven to instability by well-described photospheric motions. In most of these models the initial conditions and the photospheric driver are adjusted in such a way so that the magnetic eruption will be unavoidable (see for example [60]). However, the inability of the MHD simulations to handle simultaneously the dissipation of magnetic energy (small scales) and mechanisms for heating and acceleration (perhaps on a large scale) led many researchers to sketch the expected radiation signatures using simple cartoons. The cartoon presented by [60], for example, suggest that the high energy particles are confined in a small portion of the total volume related with the erupted structure.

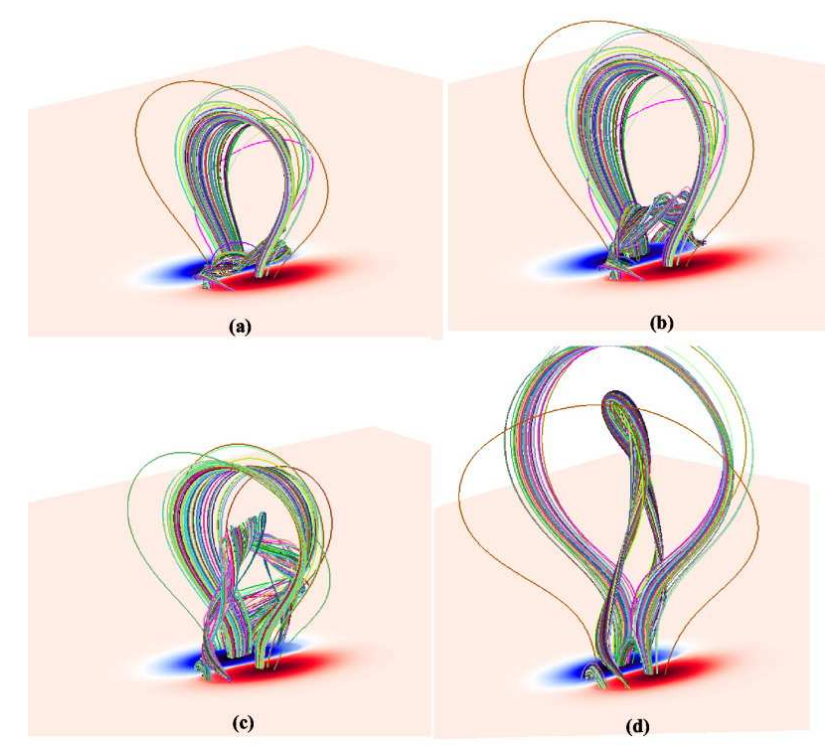

Fig. 19. Using the 3D MHD equations, even by starting from a simple magnetic geometry, the arcade is stressed and let to the eruption. There several points in this structure, were the stresses are relatively large leading to reconnection [7].

From the theoretical point of view, it is hard to prove that a huge structure with dimensions $\left[10^{10} \mathrm{~cm} \times 10^{10} \mathrm{~cm} \times 10^{5} \mathrm{~cm}\right]$ can remain stable and active for 100 s of seconds. As we have seen in Section 3.2, the dissolution of the current sheet and the formation of several smaller fragments will be its natural evolutionary path $[89,51,132]$. 
The 3D evolution of a simple photospheric magnetic field topologies leads to also to the break out model, but the magnetic topology is extremely complex [7]. The formation of a large number of tangential discontinuities (see Fig. 19) which will form numerous current filaments may be the answer to the high energy emission observed.

The simple magnetic topology for the current sheet, presented earlier and the associated simple accelerators (Direct E-field, constant flows and shocks) are probably replaced in the $3 \mathrm{D}$ magnetic topology with much more complex accelerators as we will see in the next section.

\subsection{Principal conclusions concerning models for energy release in active regions}

We outline below the main points from this section and how they influence our subsequent discussion of particle acceleration:

1. The large-scale structure: The non-linear extrapolation of observed photospheric magnetic fields gives the basic magnetic field skeleton which hosts the energy release.

2. Reconstruction of magnetic topologies: Using quite simple techniques [171], we can demonstrate many interesting properties of 3-D magnetic fields in active regions. The main themes of these approximate extrapolation are "fragmentation and self-organization", both characteristics of driven turbulent systems [171]. It is apparent that the formation of thin current sheets (TCS) in the vicinity of QSLs is the way flares start in stressed magnetic topologies [17].

3. The driver: The detailed structure and sub-Alfvénic flows of the observed photospheric magnetic field, and newly emerging magnetic flux $[64,14]$, influence the evolution and the activity of the active region. Unfortunately, detailed non linear extrapolation of photospheric magnetic fields is impossible at the present time, presenting a major drawback to our understanding of flares and CME [46].

4. Threshold for reconnection and the turbulent current sheet: Current understanding of magnetic reconnection reveals several important properties. (1) The reconnection proceeds in two modes (a) a slow mode where the TCS continues to accumulate stresses and store magnetic energy and (b) a fast mode when the TCS reaches a certain threshold when the resistivity suddenly jumps to a high value [36]. (2) The current sheet evolves to a "turbulent state" in a relatively short time (a few hundred Alfvén times) [132, 119, 98, 51].

5. Self Organized Criticality: Does the statistical behavior of flares imply that active regions are always in a Self Organized Critical (SOC) state? Several studies suggest that this can occur and is the reason behind the statistical properties of flares noted in Section 2.8. 
6. The appearance of strong turbulence during explosive phenomena: The fragmentation and self-organization of the turbulent UCS suggests that a flaring active region quickly enterers into a "turbulent state" during a flare/CME.

7. Two broad classes of flares: Stressed large-scale magnetic structures (e.g coronal loops $[128,62,63]$ ) or eruptive structures forming UCSs [7] everywhere in the stressed structure (see Fig. 19). Unfortunately the search for UCS, in analogy with the work reported for the compact loops [63], has not been performed for erupting structures or the interaction of the emerging flux with the pre-existing magnetic fields. The distributed magnetic stresses in large-scale 3-D erupting magnetic topologies remains an unexploited theoretical challenge.

The analysis presented so far in this section suggests that we have a long way to go to understand energy release in flares but many important steps have been made. The key element is: the cascade of the UCS of all scales and the inverse cascade of the coalescence of islands, form a large turbulent region (with scales of tens of thousands of kilometers) inside the evolving turbulent AR (scales tenths of millions of Kilometers). Particle acceleration mechanisms developed in the next section rely heavily on the concepts presented so far in order to build new "strong turbulent" acceleration mechanism for solar flares.

\section{Particle acceleration in turbulent electromagnetic fields}

\subsection{Brief overview of acceleration mechanisms}

Numerous books and reviews have been devoted to the challenging problem of particle acceleration $[74,164,116,166,87,168,95,122,35]$. The most prominent mechanisms analyzed in depth so far in the literature are shock waves [75, 27, 55, 44], MHD and higher frequency plasma waves [58, 120], and DC electric fields [22, 124, 125, 111].

Studying a single acceleration mechanisms mechanism (e.g. shock waves, MHD or plasma waves, DC Electric fields) in isolation implies that the energy release process favors one specific mechanism over the others. One example of where this holds is in supernova explosions when, at least in the initial stages, diffusive shock acceleration will prevail. Another example is a stable "monolithic" large-scale current sheet, where the direct electric field will dominate. However, as we discuss later, realistic models for the energy release in solar flares may have multiple acceleration mechanisms operating.

There have been a number of investigations of multiple acceleration mechanisms. Decker and Vlahos [43] analyzed Shock Drift Acceleration (SDA) when the shock was surrounded by waves. SDA is fast but not efficient, since the 
particles drifting along the electric field in the shock surface quickly leave the shock. However, the presence of MHD waves upstream and downstream of the shock sustains the acceleration process by providing a magnetic trap around the shock surface, so forcing a particle to return there many times. Thus, the particle leaves the shock surface, travels a distance $s_{i}$ inside the turbulent magnetic field, returns back to the shock surface with velocity $v_{i}$, drifts a distance $l_{i}$ along the shock electric field $E_{s c}$, changing its momentum by $\Delta p_{i} \sim e E_{s c} \cdot\left(l_{i} / v_{i}\right)$ (assuming that $\Delta p_{i}$ is small). It then escapes again, travels a distance $s_{i+1}$ before returning to the shock and drifting along the electric field: in other words, the acceleration follows a cyclic process. The process repeats itself several times before the particle gains enough energy to escape from the turbulent trap.

Let us now note some very important characteristics of this acceleration: (1) the distances $s_{i}$ traveled by the particle before returning to the shock are only indirectly related to the acceleration, since they basically delay the process and influence the overall timing, i.e. the acceleration time, an important parameter of the particle acceleration process. (2) The energy gain depends critically on the lengths $l_{i}$ that the particle drifts along the shock surface, but in a statistical sense, i.e. on the distribution of the $l_{i}, i=1,2,3 \ldots$ (3) The times $\tau_{i}$ a particle spends at the shock surface are again crucial for the energy gain, and also, together with the $s_{i}$, for the estimation of the acceleration time. (4) In the context of the total acceleration problem, i.e. the energies reached and the times needed to reach them, all three variables, $s_{i}, l_{i}, \tau_{i}$, are of equal importance.

Ambrosiano et al [8] discussed a similar problem, namely superposing a population of Alfvén waves on a current sheet. Here also the ability of the DC electric field to accelerate particles is enhanced by the presence of the MHD waves. The acceleration process is again cyclic, and is again characterized by the three variables $s_{i}, l_{i}, \tau_{i}$. The turbulent current sheet has several ways to enhance the acceleration efficiency, since the plasma inflow is dynamically driven, and causes a variety of new and still unexplored phenomena. The trapping of the particles inside the turbulent magnetic field gives rise to a new 'collision scale', and, in some circumstances, acceleration becomes dependent on an alternative 'Dreicer field', in which particle collisions are replaced by collisions with magnetic irregularities. [Indeed diffusive shock acceleration $[27,55]$ is also of a mixed type, having as elements a shock (moving discontinuity) and "converging" magnetic turbulence. Turbulence plays the role of approaching walls which scatter the particles.] In fact, it seems that most acceleration mechanisms are of a mixed type in some way. We can conclude that the mixture of mechanisms enhances the acceleration efficiency and removes some of the drawbacks attached to different, isolated mechanisms. Cyclic processes, e.g. through trapping around the basic accelerator, are important elements - if not the presupposition - of efficient and fast acceleration in space plasmas. 


\subsection{Theoretical frameworks for the study of particle acceleration}

All acceleration mechanisms in space are related to local or global plasma instabilities. The stable plasma, prior to the start of the instability, is usually assumed to be magnetized and in thermal equilibrium. In the stable plasma, the magnetic field $\boldsymbol{B}_{0}$ typically is assumed to have a simple topology, the electric field $\boldsymbol{E}_{0}$ is zero, the ambient velocity distribution is Maxwellian $f_{M}(\boldsymbol{v})$, the ambient particle density is $n_{0}$. The unstable plasma is considered as a "perturbation" of the stable state $\left(\boldsymbol{B}(\boldsymbol{r}, t)=\boldsymbol{B}_{0}+\boldsymbol{B}_{1}(\boldsymbol{r}, t), \boldsymbol{E}(r, t)=\boldsymbol{E}_{1}(\boldsymbol{r}, t)\right.$, $\left.f(\boldsymbol{r}, \boldsymbol{v}, t)=f_{M}(v)+f_{1}(\boldsymbol{r}, \boldsymbol{v}, t), n(\boldsymbol{r}, t)=n_{0}+n_{1}(\boldsymbol{r}, t)\right)$. A crucial assumption made in almost all acceleration mechanisms is that $n_{1} / n_{0}<<1$, and the energy carried by the non thermal particles is small compared the ambient energy available in the acceleration region. These assumptions are usually correct in most astrophysical systems. Solar flares and Gamma Ray Bursts (GRB) are two well documented exceptions where the accelerated particles carry a large fraction of the energy available at the accelerator.

\section{Particle dynamics in non-linear electromagnetic fields}

One important method, used by many researchers to analyze the ability of a non linear processes to accelerate particles, is the test particle approach. While this approach can give many of the important characteristics of the accelerated particles, it is based on the assumptions mentioned above, i.e. that the electromagnetic fields evolve independently of the accelerated particles. The evolution of an ensemble of non-thermal particles is determined from the calculation of the orbits of a large number of particles placed at random places inside the unstable electromagnetic fields. The equation of motion is

$$
\begin{gathered}
\frac{d \boldsymbol{p}_{i}}{d t}=q_{j} \boldsymbol{E}_{1}+q_{j}\left[\boldsymbol{v}_{i} \times\left(\boldsymbol{B}_{0}+\boldsymbol{B}_{1}\right)\right] \\
\frac{d \boldsymbol{x}_{i}}{d t}=\boldsymbol{v}_{i}
\end{gathered}
$$

where $i=1, \ldots n_{1}$ and $j$ denotes the type of particle analyzed, $\boldsymbol{p}_{i}=\gamma_{i} m_{j} v_{i}$ is the momentum, $v_{i}$ is the velocity of the particle, $m_{j}$ its mass and $\gamma_{i}=$ $\left(1-v_{i}^{2} / c^{2}\right)^{-1 / 2}$ the relativistic factor. The Lorentz force can now be divided into two parts

$$
\frac{d \boldsymbol{p}_{i}}{d t}=q_{j}\left(\boldsymbol{v}_{i} \times \boldsymbol{B}_{0}\right)+q_{j}\left[\boldsymbol{E}_{1}+\boldsymbol{v}_{i} \times \boldsymbol{B}_{1}\right]=\boldsymbol{F}_{0 i}+\boldsymbol{F}_{r i}
$$

where $F_{0}$ is forcing the particle to oscillate around the ambient magnetic field, and the $F_{r}$ is a force caused by the non-linear processes. Its behavior is so complex though that it can be modeled as a random force. Including the collisions of the non-thermal particles with the ambient plasma we get: 


$$
\frac{d \boldsymbol{p}_{i}}{d t}=\boldsymbol{F}_{0 i}-\nu \boldsymbol{p}_{i}+\boldsymbol{F}_{r i}
$$

where the collision frequency

$$
\nu \sim 10^{-11} n_{0}\left(\mathrm{~cm}^{-3}\right) / T^{3 / 2}(\mathrm{eV}) \mathrm{sec}^{-1} .
$$

Eq. 16 is a well known stochastic differential equation, introduced first by Langevin in 1908 [97]. Most known acceleration mechanisms are stochastic, since even the laminar shock or the monolithic large-scale current sheet, effectively introduce a stochastic forcing on the particles in an indirect way. We already mentioned in Sec. 4.1 that the acceleration region is finite and the particles spend a random time $\tau_{i}$ there, depending on the position they started, before escaping. Most acceleration processes known today depend critically on the characteristics of the forcing term. Multipling Eq. (16) with the momentum we derive the energy equation

$$
\frac{d E}{d t}=-\nu E+\boldsymbol{F}_{r j} \cdot\left(\boldsymbol{p}_{i} / m_{i}\right)
$$

with $E$ the kinetic energy. Following hundreds of thousand of test particles with randomly chosen initial conditions inside the acceleration region allows one to recover their statistical characteristics, i.e. injecting initially a Maxwellian distribution in random places inside the unstable plasma we may observe the evolution of the distribution function in time. The wealth of data collected from the evolution of thousands of test particles is much more accurate (but more time consuming) than the solutions of the Fokker-Planck equation which will be presented next. We will use the test particles approach extensively for the analysis of the acceleration of particles in the environment of fragmented energy release presented in Sec. 3.

\section{Fermi acceleration}

In 1949 Fermi [58] introduced a prototype stochastic acceleration mechanism to explain the acceleration of cosmic rays. His ideas were the driving force for many well known acceleration processes today, e.g. for diffusive shock or turbulent wave acceleration. Fermi chose the simplest possible random walk process in velocity space. Assuming that the "scattering centers", moving with constant speed $V$, are equally spaced (distributed at distances $L$ apart) and that the mean time between collisions is $\tau_{\text {coll }} \sim<L /(c \cos a)>\approx 2 L / c$, the mean energy gain is

$$
\left\langle\frac{d E}{d t}\right\rangle=\frac{1}{\tau_{\text {coll }}}\langle\triangle E\rangle=\frac{2 c}{3 L}\left(\frac{V}{c}\right)^{2} E=\frac{E}{\tau_{a c c}},
$$

and the mean energy gain after many interactions with the scattering centers is $\langle\Delta E\rangle=(4 / 3)(V / c)^{2} E[58]$. Collisions between particles are ignored but 
particles escape from the spatially restricted acceleration region in a characteristic time $\tau_{e s c}$. The simplest way to generalize the ideas of Fermi is the well known "Fermi map" [103], where

$$
\begin{aligned}
& v_{n+1}=v_{n}+2 x_{0} \omega \sin \omega t_{n} \\
& t_{n+1}=t_{n}+\frac{2 L}{v_{n+1}}
\end{aligned}
$$

representing a ball moving between two parallel plates (see Fig. 20). The

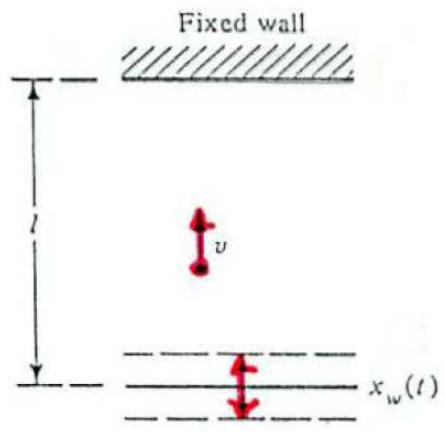

Fig. 20. The ball is moving between the walls. The lower wall is oscillating with frequency $\omega$ and amplitude $x_{0}$. The walls are separated by a distance L.[103]

kick $\Delta v$ in the $n^{\text {th }}$ step is a periodic function of time, and the time between collisions is inversely proportional to the velocity. The change of the energy is instantaneous.

\section{Diffusion Equations}

The statistical evolution of a large number of particles inside a collection of equally spaced and slowly moving "scattering centers" can be discussed, under certain constraints, with the use of the Fokker-Planck equation. Particles moving inside stochastic fields follow very complicated orbits. The particles perform, depending of the structure of the fields, strange "walks" inside a turbulent electromagnetic medium. The simplest example is the Brownian particle executing a "random walk", and it represents the motion of a "heavy" particle inside a gas of particles that is in equilibrium. Eq. (16) can handle these problems when the random force obeys a Gaussian distribution.

Assuming that every step of the "walk" is totally independent (a Markovian process), we can derive formally the Fokker-Planck equation, which has been an important instrument for the study of high energy particles in astrophysics $[129,147]$. The derivation of the Fokker-Planck equation is beyond 
the scope of the present review but it is important to stress that it remains an accurate representation only of phenomena remaining close to equilibrium, and when the forcing term is very weak. It represents a slowly evolving distribution of particles experiencing a weak and rapidly oscillating force. The simplest form of the Fokker-Planck Equation is the one describing the energy diffusion (assuming that the particles remain always isotropic, i.e. the scattering process is so frequent that it manages to sustain isotropy all the time):

$$
\begin{aligned}
\frac{\partial n_{1}(E, t)}{\partial t} & =\frac{\partial^{2}}{\partial E^{2}}\left(D_{E E}(E) n_{1}(E, t)\right)-\frac{\partial}{\partial E}\left[\left(\left\langle\frac{d E}{d t}\right\rangle-\left(\frac{d E}{d t}\right)_{l}\right) n_{1}(E, t)\right] \\
& -\frac{n_{1}(E, t)}{\tau_{e s c}(E)}+Q(E, t)
\end{aligned}
$$

where the term $<d E / d t>$ represents the systematic acceleration,

$$
D_{E E}(E)=\frac{<E(t)^{2}>}{t}
$$

is the diffusion coefficient in energy space [37], $(d E / d t)_{l}$ represents the energy losses due to Coulomb collisions and radiation, $\tau_{\text {esc }}(E)$ is the energy dependent loss rate of particles out of the finite acceleration system and $\mathrm{Q}(\mathrm{E})$ represents the replenishment of particles inside the accelerator (usually taken as a Maxwellian distribution times an injection rate).

Fermi solved Eq. 21 in its simplest form. Assuming that $\langle d E / d t>$ is given by Eq. (18) and assuming that: (1) losses are not important, (2) no source term is included, (3) there is no diffusion in energy (but only systematic acceleration) and searching for a steady state solution, he found:

$$
\frac{d n_{1}(E)}{d E}=-\left(1+\frac{\tau_{a c c}}{\tau_{e s c}}\right) \frac{n_{1}}{E} .
$$

The solution is well known,

$$
n_{1}(E) \sim E^{-r}
$$

where $r=1+\tau_{a c c} / \tau_{e s c}$. The solution obtained from the Fokker-Planck equation predicts, in the case of cosmic rays, the correct observed functional form, but the index $r$ is not in agreement with the observations. More sophisticated results can be reached by assuming a spectrum of MHD waves and incorporating the terms dropped by Fermi [161, 96, 4, 139]. Using quasilinear theory, we can also incorporate wave generation, cascade, and dissipation processes [121] and create a self consistent system of equations. The Fokker-Planck equation is a useful tool for the analysis of particle diffusion in space and energy, but it is very restricted concerning the kind of turbulent environments it can handle. The assumptions behind Eq. 21 present a barrier to the analysis of the systems appearing during the turbulent flare model presented earlier.

Before closing this section it is worth mentioning a few more points. 
- How can we estimate the transport coefficients (if it is not possible to derive analytical expressions) using Eq. 16? Following the orbits of many particles and using Eq. 16 , we can estimate numerically the systematic acceleration $<d E / d t>$ term and the transport coefficient $D_{E E}$.

- Is diffusion always normal (Brownian motion is the prototype for normal diffusion)? The spatial diffusion of a Brownian particle inside a gas in equilibrium follows a simple law $\left\langle x^{2}\right\rangle=D t$, and this type of diffusion is called normal [21]. In a turbulent plasma the diffusion processes are much more complicated (they follow strange kinetics in fractal media) and give the relation $\left\langle x^{2}>\sim t^{a}\right.$ where the index $a$ can be smaller than one (sub-diffusion) or larger than one (super diffusion) $[176,123]$.

- If the diffusion is not normal, is the Fokker-Planck equation still valid? Unfortunately the Fokker-Planck equation is invalid when the diffusion processes are anomalous. More complicated partial differential equations are needed and the derivation and the solution of such equations are not as easy as the standard Fokker-Planck equation. Using more sophisticated numerical methods, e.g. Monte Carlo [69] simulations or the fractional kinetics approach $[176,123]$, we can obtain more realistic results.

\section{Monte-Carlo simulations}

A numerical technique, used widely in many astrophysical problems and which will be extremely useful for the results presented in the next section, is the Monte-Carlo approach. It is instructive to present this approach in a way that can be used in the context of particle acceleration [69, 126].

Let us assume that a particle starts at a given point $\boldsymbol{x}_{0 i}(t=0)$ in space (this point is randomly selected inside the acceleration region) and with initial velocity $\boldsymbol{v}_{0 i}(t=0)$. The initial velocities are selected from a sample which follows a Maxwellian distribution (so the bulk of the particles are in equilibrium initially). The next step is to move the particle a distance $\Delta_{i}$ till the next "scattering center" i.e. $x_{\text {new }}=x_{\text {old }}+\Delta_{i}$, along this path is reached, when the particle looses energy either by collisions or by radiation losses and arrives at the new position with a new velocity, estimated as $v_{\text {new }}=v_{\text {old }}-\nu_{\text {loss }} \Delta_{i}$. At the new point the particle enters a "scattering center", gains or loses energy, and departs with a new velocity $v_{\text {new }}=v_{\text {old }} \pm \Delta_{i}$. The time has evolved as $t_{\text {new }}=t_{\text {old }}+\frac{\Delta_{i}}{v_{\text {new }}}+\Delta T_{\text {scat }}$. We assume that inside the localized scatterer the particle follows a complicated trajectory (which we do not follow in detail). The particle stops moving when its position is outside the limits of the acceleration region, or the energy release time is shorter than $t_{\text {new }}$. Applying all the above to Fermi acceleration is simple since all $\Delta_{i}$ are equal $\left(\Delta_{i}=L\right)$, no losses are included, the particles spend no time in the scattering center $(\Delta T=0)$ and $\Delta v_{i}$ is given by a simple formula (see the Fermi map).

In more complex environments, the three unknown variables $\Delta_{i}, \Delta v_{i}, \Delta T_{i}$ are considered random and distributed according to probability distributions 
that should incorporate the statistical properties of the system under consideration. Monte Carlo simulations are a very useful and flexible tool to treat these systems. We will outline a specific example of the Monte Carlo method in solar flares in Sec. 4.6.

\subsection{Turbulent current sheets as particle accelerators}

According to our understanding of magnetic reconnection, several potential mechanisms for particle acceleration co-exist at an UCS. Plasma flows driving turbulence, shock waves, and DC electric fields are expected to appear simultaneously inside and around a driven and evolving UCS. If the UCS is located in the middle of a turbulent magnetic topology, all these phenomena will be enhanced and the sporadic external forcing of the plasma inflow into the UCS will create bursts of sporadic acceleration.

Analyzing the orbits of particles in an isolated current sheet is a very interesting problem and the non-linear characteristics of the trajectories are impressive (see [54] and references therein). Most studies reported so far use analytical solutions of the static electromagnetic fields for the reconnecting current sheets in 2 -D or 3 -D $[152,127,106,179,180,41,42]$. We feel that these studies are interesting but bear little resemblance with the dynamic evolution of the turbulent UCS discussed earlier in Sec. 3.2 where the main emphasis was shifted towards the interaction of particles with smaller scale structures within the current sheet $[88,52,133]$. The nonlinear evolution of the UCS is characterized by the formation of small scale structures, especially in the lateral regions of the computational domain, and coalescence of magnetic islands in the center. This behavior is reflected in the three-dimensional structure of the electric field, which shows that the initial equilibrium is destroyed by the formation of current filaments.

Kliem [88] started off with a 2-D analytical description of the magnetic field topology, which includes two colliding islands. The electric field is derived from the coalescence of the islands moving with characteristic speed $\mathbf{u}$. The acceleration is due to the convective electric field $E_{\text {conv }} \sim-\mathbf{u} \times \mathbf{B}$ and happens at the $\mathrm{X}$-line. Electrons reach relativistic energies in a very short time as they move inside these electric fields. Drake et al. [52] also discuss the interaction of particles with contracting magnetic islands. An attempt to draw the analogy with Fermi acceleration was also made and an estimate of the systematic acceleration was:

$$
\left\langle\frac{d E_{\|}}{d t}\right\rangle=-\frac{E_{\|}}{\tau_{a c c}}
$$

where

$$
\tau_{a c c}=2 \frac{u_{x}}{\delta_{x}} \frac{B_{x}^{2}}{B_{0}^{2}}
$$

and $2 \delta_{x}$ is the length of the island, $u_{x}$ the velocity of the contracting island (of order the Alfvén speed), $B_{x}, B_{0}$ the reconnecting and the ambient magnetic 
field respectively. Several interesting conclusions were reached e.g. particles interacting with many islands can easily reach relativistic energies, and the particle distribution (obtained by solving a simplified form of the diffusion equation) was tending towards a power law with index around -1.5 for solar parameters. Onofri [133] used the resistive 3-D MHD equations (see Sec. 3.6) to analyze the evolution of a perturbed UCS and the electric field derived from ohms law $\mathbf{E} \sim \eta \mathbf{J}-\mathbf{v} \times \mathbf{B}$. Fig. 21 shows the isosurfaces of the electric field at different times calculated for two different values of the electric field: the red surface represents higher values and the blue surface represents lower values. The structure of the electric field is characterized by small regions of space where the field is stronger, surrounded by a larger volume occupied by lower values. At later times the fragmentation is more evident, and at $t=400 \tau_{A}$ (where $\tau_{A}$ is the Alfvén time), the initial current sheet has been completely destroyed and the electric field is highly fragmented. The strong electric field regions are acceleration sites for the particles and their distribution in space fills a larger portion of the simulation box at later times, with increasing possibility to accelerate a higher number of particles.

To give a measure of the fragmentation of the electric field, we calculated the fractal dimensions of the fields shown in Fig. 21, using the box-counting definition of the fractal dimension [133]. The magnitude $E$ of the electric field at each gridpoint of the simulation domain was estimated, and the distribution function of these quantities was constructed (see Fig. 22 for $t=50 \tau_{A}$.) We separately plot the resistive and the convective component of the electric field. The resistive part is less intense than the convective part, but it is much more important in accelerating particles, as we verified by performing some simulations where only one of the two components was used. Protons and electrons are injected into the simulation box where they move under the action of both components (convective and resistive) electric field and magnetic fields, which do not evolve during the particle motion. This is justified by the fact that the evolution of the fields is much slower than the acceleration process, electrons and ions are accelerated on a short time scale to very high energies, and in such short times the fields would not change significantly according to the MHD simulation.

The trajectories of the test particles inside the box are calculated by solving the relativistic equations of motion, using a fourth order Runge Kutta adaptive step-size scheme. Since the magnetic and the electric fields are given only at a discrete set of points (the grid-points of the MHD simulation), both fields are interpolated with local three-dimensional linear interpolation to provide the field values in between grid-points.

For the case of electrons, the particles' energy distribution at different times is shown in Fig. 23. Some of the test particles are quickly accelerated to high energies so that the initial Maxwellian distribution changes, developing a tail that grows in time. The kinetic energy of the electrons increases very rapidly, and in a short time it equals the energy contained in the magnetic field. Since there is no back reaction of the particles onto the fields, there is no 


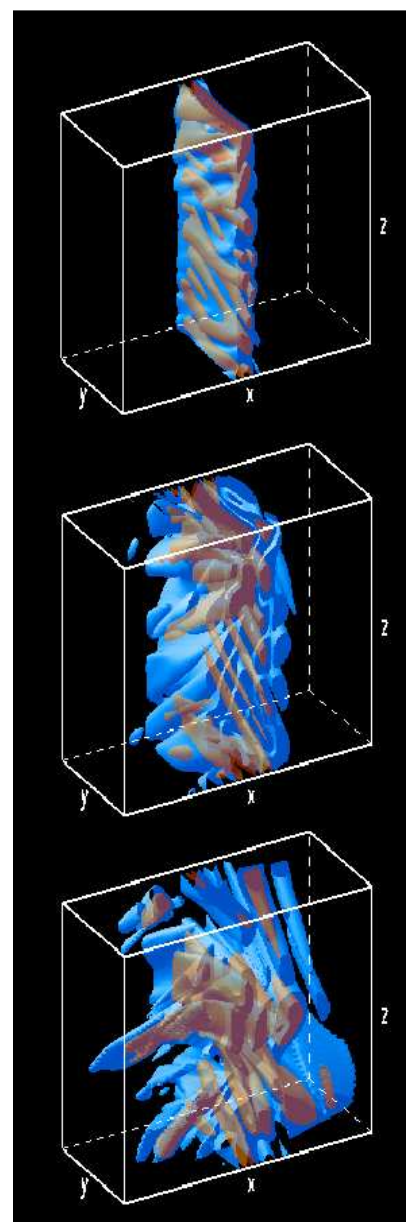

Fig. 21. Electric field isosurfaces at $t=50 \tau_{A}, t=200 \tau_{A}$ and $t=400 \tau_{A}$. [133]

limit to the energy they can gain until they leave the simulation box. For this reason the particle motion was followed only as long as their energy is still less than $50 \%$ of the magnetic field energy $W_{B}$, which is up to $t_{p e}=8 \times 10^{-5} s$.

The maximum kinetic energy at the end of the run turns then out to be about $1 \mathrm{MeV}$. Collisions are not included in the simulations because the collisional time is about $t_{c}=5.5 \times 10^{-3} \mathrm{~s}$, which is much longer than $t_{p e}$. In the final distribution, the logarithmic slope of the power law tail is $\simeq 1$. The power law tails of the distributions start at an energy of about $1 \mathrm{KeV}$. The total number of particles contained in the tail of the distributions $\left(E_{K} \geq 1\right.$ $\mathrm{keV}$ ) is $\simeq 6 \times 10^{37}$ for the assumed values of the particle density $n_{0}$ and length $l_{x}$. Below $1 \mathrm{keV}$, the electrons have a thermal distribution. 


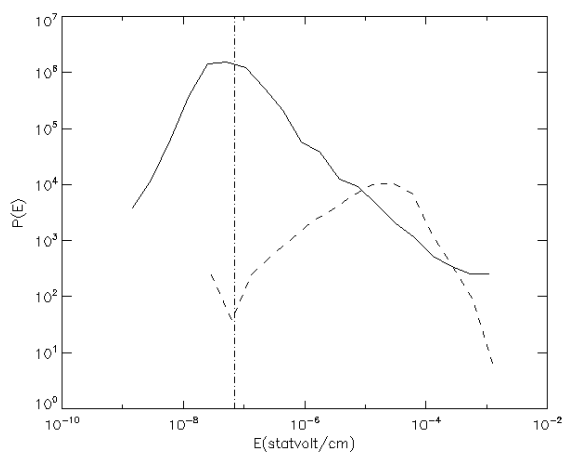

Fig. 22. Distribution function of the resistive (dashed line) and convective (solid line) electric field at $t=50 \tau_{A}$. The vertical line represents the value of the Dreicer field in the solar corona. [133]

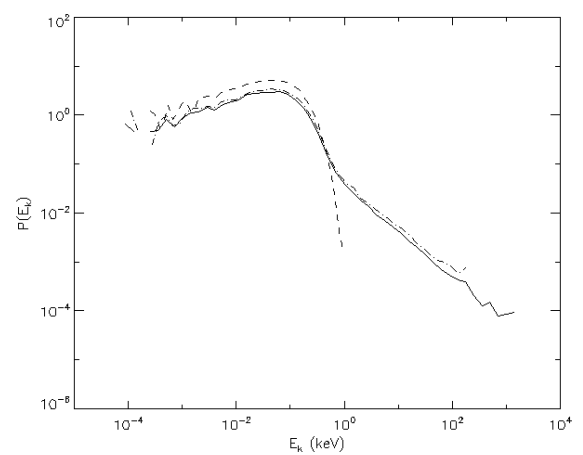

Fig. 23. Distribution function of electron kinetic energy at $t=8 \times 10^{-5} \mathrm{~s}$ (solid line), $t=3 \times 10^{-5} \mathrm{~s}$ (dotted-dashed line) and the initial distribution (dashed line). The electromagnetic field is given at $t=72 \mathrm{~s}$.[133]

Turning to protons, Onofri et al. find that acceleration is much less efficient than for electrons, only at $t_{p i}=3 \times 10^{-3} s$ do they reach a maximum kinetic energy of about $1 \mathrm{MeV}$, with energy distributions that are similar to those of the electrons. Because of the much slower acceleration time scale of the protons, the time limit for our simulation is determined by the electrons, $t_{p e}$ $\left(t_{p e}<<t_{p i}\right)$ - at times as large as $t_{p i}$, the electrons would have absorbed all the available magnetic energy $W_{B}$. At the time limit $t_{p e}$ then, the distribution of the ions has remained close to the initial Maxwellian, with just minor gain in energy.

The results of these simulations show that a decayed and fragmented current sheet can be a very efficient accelerator. The particles absorb a large amount of energy from the magnetic field in a short time, and the magnetic 
and electric fields lose a large fraction of their energy. However, the backreaction of the particles is not taken into account in the test particle simulations reported here, which in that sense are not self-consistent. Our results suggest that the lifetime of a current sheet of this size in the solar corona is very short since energetic particles absorb a large fraction of the available magnetic energy. As a consequence of the back-reaction, the magnetic and electric field would change more quickly than the MHD simulation shows, the acceleration process can be expected to be slower, and the resulting energy distributions will probably be different.

The limitations of this approach, in the case of the electrons, are reflected in the inability of our results to reproduce all the characteristics of the distributions that are observed in solar flares (e.g. variation of power-law slopes). In the case of the ions, the situation is different. Their maximum energy at the time-limit of our simulations is lower than the energy that protons usually reach during solar flares.

We can thus conclude that ions are not accelerated to the high energies observed during solar flares by single, isolated, turbulent current sheets. Stressed and complex large-scale magnetic topologies can though form simultaneously many current sheets [63], and it has been shown that the interaction of the ions (and electrons) with many current sheets can be a very efficient accelerator $[159,160]$.

\subsection{Acceleration in stressed magnetic fields}

The model for the compact flare analyzed in Section 3.3 is used to examine the acceleration of particles $[159,160]$. Many of the techniques used are similar to those in the previous section. Here the MHD simulation was performed on a numerical grid with 200 points between the photospheric boundaries (the $\mathrm{x}$-coordinate), and 60 points in each of the transverse directions (the $\mathrm{y}$ - and z-coordinates). Only the coronal portion of the magnetic field is considered, so that in terms of a dimensionless length, the electric and magnetic fields are confined to $\mathbf{L}=\left(L_{x}, L_{y}, L_{z}\right)=(1.6,0.1,0.1)$. Physical lengths are obtained by multiplying $\mathbf{L}$ by a factor $\mathbf{L}$. Most of the results presented use $L=10^{9}$ $\mathrm{cm}$. The value of the background coronal density is taken to be $10^{10} \mathrm{~cm}^{-3}$, the initial background magnetic field is taken to be $B=100 \mathrm{G}$ and the coronal plasma beta is equal to 0.04 . The electric field (both resistive and inductive) arising in this model, and their potential as particle accelerators was analyzed. The inductive field appeared to be negligible (especially for high energy particles) because its component parallel to the magnetic field is zero. The resistive electric field is distributed over the domain in the form of a hierarchy of current sheets. Fig. 24 shows the snapshot of these current sheets from the MHD model that is used in this paper. It is obvious that a very complex topology is formed. In between the current sheets no electric field exist, while the electric field inside the current sheets takes on values between 
$\pm 310^{-2}$ statvolt $/ \mathrm{cm}$. The average absolute value of the electric field is $510^{-4}$ statvolt $/ \mathrm{cm}$.

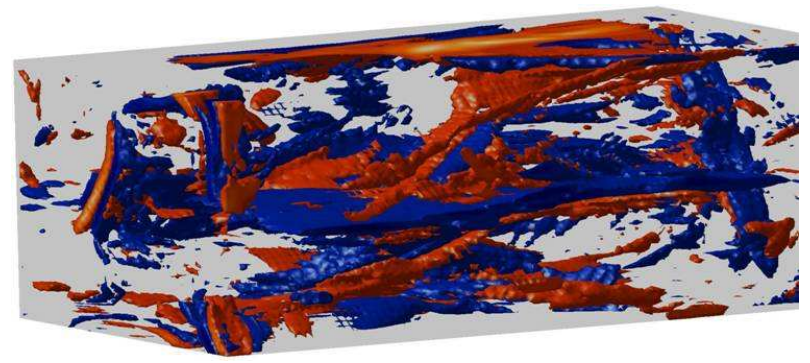

Fig. 24. Snapshots of the resistive electric field configurations within the coronal volume, as calculated from the global MHD model. The blue and red regions represent electric field regions that point towards the left and right foot points respectively. The details of the model are described in the text [159].

The output of the MHD model, specifically the three-dimensional electric and magnetic fields, are used as a basis for studying particle acceleration. Particles were tracked in frozen fields using a similar numerical scheme to that in the previous section, the frozen field being justified by the separation of timescales for acceleration $(<1 \mathrm{sec})$ from the characteristic coronal evolution time ( $>1 \mathrm{sec})$. Acceleration was considered only in the coronal part of the model, so current sheets appearing at the foot points were ignored.

In each example discussed, 30,000 particles are injected with an initial Maxwellian distribution with a temperature of $1.2 \times 10^{6} \mathrm{~K}$. The initial positions and pitch-angles of the particles are random. The particles are injected in the MHD domain simultaneously and are considered as "lost" when they leave the simulation box and are not replaced. Again, feedback is not included.

In Figure 25a a 1-D sample of the x-component of the electric field along the domain is shown. The distribution function of the values of the magnitude of the electric field is shown in Figure 25b. The distribution has a power law component with an index value of -2.8 which terminates at a cut-off at the highest values.

Figure 26 shows the final distribution function at $\mathrm{t}=0.5 \mathrm{~s}$. The energy used to construct the distribution is either their final energy, or that with which they left the domain. This distribution function has three main parts, the thermal part, and the two power law components with indexes equal to -0.7 and -2.7 respectively. This distribution is in fact comprised of a number of "classes" of particles which behave differently throughout the simulation. In particular, particles can leave the domain through either the sides, or the ends, or become confined to the corona with or without energisation.

The stressed coronal fields are a very effective particle accelerator, with both electrons and protons attaining relativistic energies in a very short time 

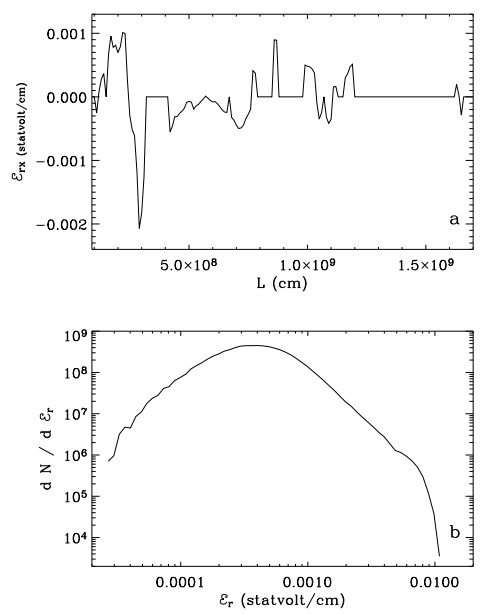

Fig. 25. (a) An arbitrary 1-D sample of the resistive electric field along the domain (the x-direction) (b) Distribution function of the resistive electric field. [160]

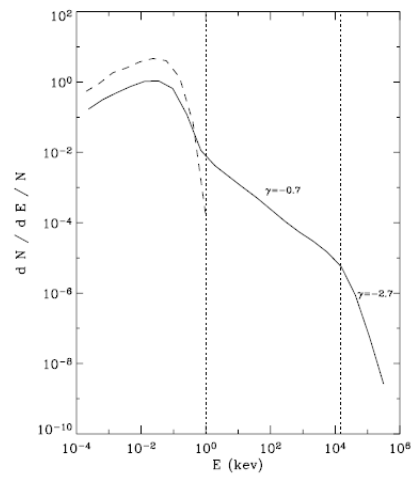

Fig. 26. Distribution function for all the particles injected in the domain at $t=0$ (dashed curve) and at the end of the run (solid curve). $\gamma$ is the power-law index value. [160]

throughout the corona: for example electrons are accelerated to relativistic energies in milli-seconds. The acceleration appears to have four phases, with the maximum energies rising, peaking and then decaying, as well as there being an extended acceleration phase lasting for almost $1 \mathrm{sec}$. The energy reached scales with the coronal length scale.

Combining the fragmentation of a single current sheet discussed in the previous section and the results presented here, it easy to conclude that the solar corona forms a multi-scale environment, starting from UCS with characteristic length $>10^{9} \mathrm{~cm}$, which cascade to very small structures of the order 
of $100 \mathrm{~s}$ of meters. At the same time the unstable current sheets may force other TCSs to go unstable, therefore the particle dynamics become extremely complicated. Unfortunately no current code can handle so much complexity and we have to use approximate methods.

\subsection{Particle acceleration by MHD turbulence}

\section{Low amplitude waves $(\delta B / B<<1)$}

When accelerating particles by MHD waves one considers the Alfven branch for ions since it has a resonance below the proton gyrofrequency $\Omega_{H}$, and the fast mode branch, (magnetosonic or whistlers), for electrons which has a resonance below the electron gyrofrequency $\Omega_{e}$. The analysis of the interaction of particles and waves was initially based on the assumption that a large volume was filled with low amplitude MHD waves, with a power law spectrum $W(k) \sim k^{-q}$. The entire acceleration volume was constantly replenished and the distribution remained isotropic. With these assumptions, the FokkerPlanck equation remains relatively simple (see Eq. 21) and in order to make thing even simpler, the time evolution of the accelerated particles was ignored. Recently several attempts were made to improve the above scenario and the spectral evolution of the waves was included [121]. This would appear to be an even more complicated project since the wave-wave interactions included can hardly capture the fragmentation of the energy release presented in the previous section [140].

The main assumption in these studies is that large-scale current sheets will produce low amplitude, long wavelength MHD waves (e.g. Alfven waves), which will cascade to smaller and smaller scale MHD waves, until they will reach the dissipation scale. It is clear that all these processes will be highly anisotropic in space and time. Damping of the waves is also anisotropic and this will modify the cascade process [140].

We can then conclude that if reconnection is the main mechanism for the energy release in solar flares, attempts to reduce the energy release process to an extremely simplified coupled system of low amplitude, homogeneous MHD waves which evolve quasi-linearly, does not resembles a real flare, especially when $50 \%$ of the magnetic energy goes to high energy particles.

\section{Large amplitude waves $(\delta B / B \geq 1)$}

Dmitruk et al. [47, 48] analyzed the acceleration of particles inside 3-D MHD turbulence. The compressible MHD equations (see Eqs. 4-9) were solved numerically. In these simulations the decay of large amplitude waves was studied. After a very short time (a few Alfvén times) a fully turbulent state with a broad range of scales has been developed (Fig. 27).

As in previous sections, the magnetic field is directly obtained from the numerical solution of the MHD equations with an electric field derived from 

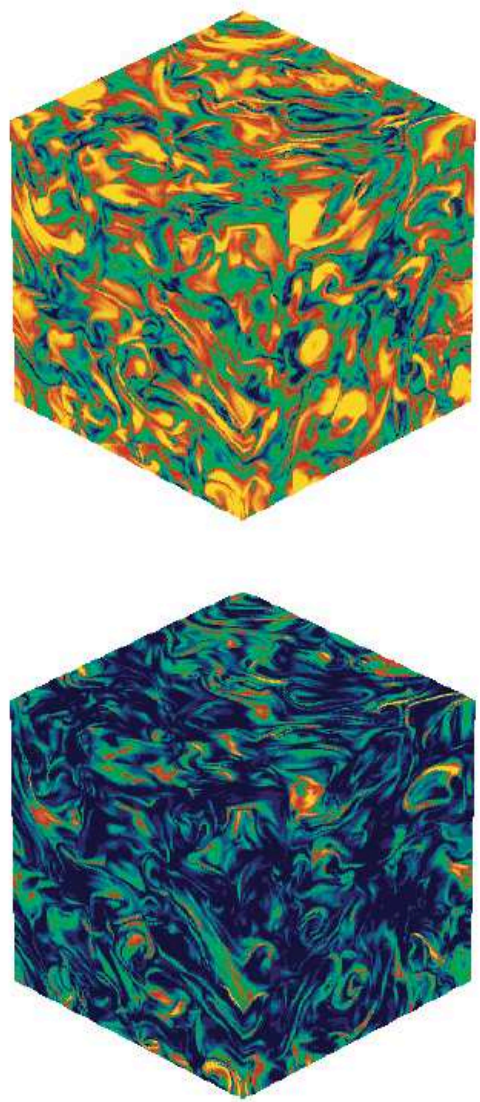

Fig. 27. Visualization of the turbulent magnetic field $|B|$ (top) and electric field $|E|$ (bottom) in the simulation box. High values are in yellow (light) and low values in blue (dark)[47]

Ohm's law (see Eq. 10). It is obvious that the electric field is an intermittent quantity with high values observed in less space filling distribution. Magnetic and electric fields show a broad range of scales and high degree of complexity. The energy spectrum of the MHD fields is consistent with a Kolmogorov- $5 / 3$ power law. The structure of the velocity field and the current density along the external magnetic field $\left(J_{z}\right)$ can be seen in Fig. 28 The formation of strong anisotropies in the magnetic field, the fluid velocity and the associated electric field is observed. The overall picture is that current sheet structures along the DC field are formed as a natural evolution of the MHD fields.

Following thousands of particles particles inside the simulation box we can learn many of the statistical properties of their evolution, e.g. $\sqrt{\left\langle\Delta x^{2}\right\rangle}$, $\sqrt{\left.<\Delta v^{2}\right\rangle}$, the velocity distribution etc. Electron and ions are accelerated 


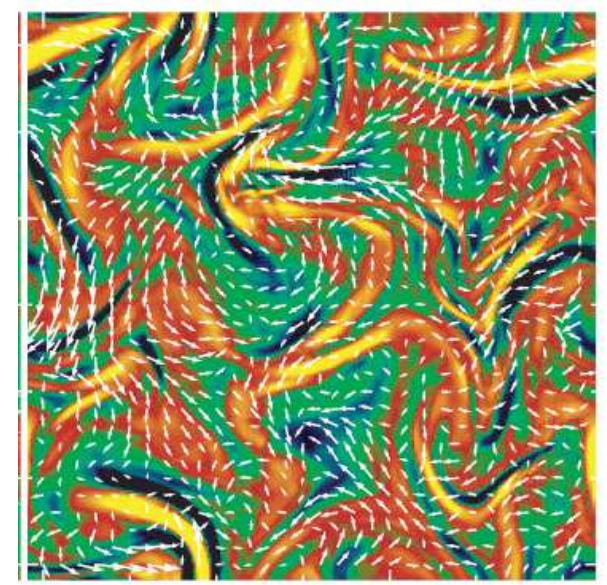

Fig. 28. Cross section of the current density along the external magnetic field in color tones. Yellow (light) is positive $J_{z}$, blue (dark) is negative and the superposed arrows for the velocity field[48]

rapidly, and the non-thermal tails form power law distributions. Most particles seem to escape the volume by crossing only a few of the randomly appearing current sheets. A few particles are trapped in these structures and accelerated to very high energies. The Fokker-Planck equation is not the appropriate way to capture the random appearance of coherent structures inside such a turbulent environment.

Arzner and Vlahos [15] investigated the effect of multiple localized resistive spots on coronal particle acceleration. They considered collisionless test particles in evolved homogeneous MHD turbulence with electromagnetic fields modeled by

$$
\begin{aligned}
& \mathbf{B}=\nabla \times \mathbf{A} \\
& \mathbf{E}=-\partial_{t} \mathbf{A}+\eta(\mathbf{j}) \mathbf{j},
\end{aligned}
$$

where $\mu_{0} \mathbf{j}=\nabla \times \mathbf{B}$ and $\eta(\mathbf{j})=\eta \theta\left(|\mathbf{j}|-j_{c}\right)$ is an anomalous resistivity switched on above the critical current $j_{c} \sim e n c_{s}$ [134]. Here $c_{s}(n)$ is the sound speed (number density) of the background plasma. The vector potential $\mathbf{A}(\mathbf{x}, t)$ is modeled as a random field, subject to the MHD constraints

$$
\mathbf{E} \cdot \mathbf{B}=0 \text { if } \eta=0 \text { and } E / B \sim v_{A} .
$$

Eq. 27 can be satisfied in several ways. A spectral representation in axial gauge, $\mathbf{A}(\mathbf{x}, t)=\sum_{\mathbf{k}} \mathbf{a}(\mathbf{k}) \cos \left(\mathbf{k} \cdot \mathbf{x}-\omega(\mathbf{k}) t-\phi_{\mathbf{k}}\right)$ with $\mathbf{a}(\mathbf{k}) \cdot \mathbf{v}_{A}=0$ and dispersion relation $\omega(\mathbf{k})=\mathbf{k} \cdot \mathbf{v}_{A}$, which is an exact solution of the induction equation with a constant velocity field $\mathbf{v}_{A}$ was used. For simplicity, $\mathbf{A}(\mathbf{x}, t)$ is taken as Gaussian with random phases $\phi_{\mathbf{k}}$ and (independent) Gaussian amplitudes $\mathbf{a}(\mathbf{k})$ with zero mean and variance 


$$
\left\langle|\mathbf{a}(\mathbf{k})|^{2}\right\rangle \propto\left(1+\mathbf{k}^{T} \mathbf{S} \mathbf{k}\right)^{-\nu} .
$$

A constant magnetic field $B_{0}$ along $\mathbf{v}_{A}$ can be included without violating Eq. 27. The total MHD wave velocity is $v_{A}^{2}=\mathcal{B}^{2}\left(\mu_{0} \rho\right)^{-1}$ with $\mathcal{B}^{2}=B_{0}^{2}+$ $\sigma_{B}^{2}$ and $\sigma_{B}^{2}=\frac{1}{2} \sum_{\mathbf{k}}|\mathbf{k} \times \mathbf{a}(\mathbf{k})|^{2}$ the magnetic fluctuations. The matrix $\mathbf{S}=$ $\operatorname{diag}\left(l_{x}^{2}, l_{y}^{2}, l_{z}^{2}\right)$ in Eq. (28) contains the outer turbulence scales, and the index $\nu$ determines the regularity of the two-point function at short distance. The presented simulations have $\nu=1.5, \mathbf{v}_{A}=\left(0,0, v_{A}\right)$, and one turbulence scale is longer by an order of magnitude than the two (equal) others, which describes migrating and reconnecting twisted flux tubes).

The vector potential contains some hundred wave vectors in the inertial shell $\min \left(l_{i}^{-1}\right)<|\mathbf{k}|<10^{-2} \cdot r_{L}^{-1}$ with $r_{L}$ the rms thermal ion Larmor radius. We focus on strong turbulence $\left(\sigma_{B} / B_{0}>1\right)$. The rms magnetic field $\mathcal{B}$ is a free parameter, which defines the scales of the particle orbits. The localized enhancement of the resistivity will: (1) enhance the local heating inside the unstable current sheet, $Q_{j}=\eta_{j} j^{2}$ forming what we will call here "hot spots" and (2) dramatically enhance the particle (ion and electron) acceleration. The fast heat transport away from the hot plasma will soon transform them into hot loops.

The physical units used in this study [15] are selected to represent the solar atmosphere. In SI units and for typical values $\mathcal{B} \sim 10^{-2} \mathrm{~T}, n \sim 10^{16} \mathrm{~m}^{-3}$, $T \sim 10^{6} \mathrm{~K}$, the reference scales are as follows (electron values in brackets): time $\Omega^{-1} \sim 10^{-6} \mathrm{~s}\left(6 \cdot 10^{-10} \mathrm{~s}\right)$; length $c \Omega^{-1} \sim 300 \mathrm{~m}(0.17 \mathrm{~m})$; thermal velocity $\sim 1.2 \cdot 10^{5} \mathrm{~ms}^{-1}\left(5 \cdot 10^{6} \mathrm{~ms}^{-1}\right)$; sound speed $c_{s} \sim 1 \cdot 10^{5} \mathrm{~ms}^{-1}$; Alfvén speed $v_{A} \sim 2 \cdot 10^{6} \mathrm{~ms}^{-1}$; electron-ion collision time $\tau \sim 0.003 \mathrm{~s}$; Dreicer field $E_{D}=n e^{3} \ln \Lambda /\left(4 \pi \epsilon_{0}^{2} k T_{e}\right) \sim 3 \cdot 10^{-2} \mathrm{Vm}^{-1}$. Time is measured in units of $\Omega^{-1}=m / q \mathcal{B}$; velocity is measured in units of the speed of light; distance is measured in units of $c \Omega^{-1}$.

When an initially Maxwellian population is injected into the turbulent electromagnetic field given by Eqs. (25)-(26), the particles can become stochastically accelerated. Due to their large inertia, protons gain energy in relatively small portions. This is not so for electrons. The momentum evolution of collisionless electrons of the high-energy tail of a Maxwellian is shown in [15] (see Fig. 4 in [15]).

Since electrons have much smaller Larmor radius, they follow the field lines adiabatically and gain energy only when dissipation regions are encountered. The resulting orbits then have large energy jumps, so that a Fokker-Planck description is inappropriate [147].

We can then conclude that a large-scale turbulent cascade leads to highly anisotropic structures randomly placed inside the acceleration volume. Electrons and ions evolve inside these structures forming power law energy distributions, and the acceleration time is relatively short. The Fokker-Planck equation is invalid inside such environment and the sudden random formation of DC electric fields along the external magnetic fields is apparent. In these simulations current sheets are part of the turbulent cascade processes. 
Collisions were not include in any of the studies reported above [47, 15, 48] therefore they are applicable on the corona $\left(n=10^{9} \mathrm{~cm}^{-3}, T \sim 100 \mathrm{eV}\right)$ where the acceleration time is usually milliseconds, much faster than collisional losses.

\subsection{Particle acceleration in complex magnetic topologies}

Our aim in this chapter is to take advantage of the properties of isolated UCS as accelerators, but at the same time to incorporate the fact that the dissipation happens at multiple, small-scale sites. Many attempts have been made in the past to analyze the evolution of a distribution of particles inside a collection of nonlinear dissipation structures $[9,10,11]$. We will attempt here to present all these developments in a more unified way [172].

The 3-D magnetic topology, driven from the convection zone, dissipates energy in localized UCS, which are spread inside an active region, providing a natural fragmentation for the energy release and a multiple, distributed accelerator. In this way, the large-scale magnetic topology acts as the backbone which host the UCS, and the spatio-temporal distribution of the latter defines the type of flare, its intensity, the degree of energization and acceleration of the particles, the acceleration time-scales etc. Evolving large-scale magnetic topologies provide a variety of opportunities for acceleration which is not restricted to the impulsive phase, but can also take place before and after it, being just the manifestation of a more relaxed, but still driven topology. Depending on the extent to which the magnetic topology is stressed, particles can be accelerated without a flare, and even long-lived acceleration in nonflaring active regions must be expected. Consequently, the starting point of the model to be introduced below is a driven 3-D magnetic topology, which defines a time-dependent spatial distribution of UCS inside an active region. The details of the mechanisms involved in the acceleration of particles inside the UCS are not essential in a stochastic modeling approach.

Since the global characteristics of the energy release play a crucial role in the acceleration of particles, it is important to make use of the new developments in the theory of SOC models for flares. Also taken into account are ideas from the theory of Complex Evolving Networks [5, 49, 50], adjusted though to the context of plasma physics: the spatially distributed, localized UCS can be viewed as a network, whose 'nodes' are the UCS themselves, and whose 'edges' are the possible particle trajectories between the nodes (UCS). The particles are moving around in this network, forced to follow the edges, and undergo acceleration when they pass by a node. The network is complex in that it has a non-trivial spatial structure, and it is evolving since the nodes (UCS) are short-lived, as are the connectivity channels, which even change during the evolution of a flare. This instantaneous connectivity of the UCS is an important parameter in our model: it determines to what degree multiple acceleration is imposed onto the system, which in turn influences the instantaneous level of energization and the acceleration time-scale of the particles. 
The UCS are short lived and appear randomly inside the large-scale magnetic topology when specific conditions for instability are met. Modeling this dynamic accelerator requires the knowledge of three probability density functions [172]:

- The probability density $P_{1}(s)$ defines the distribution of the distances a charged particle travels freely in between two subsequent encounters with a UCS. The series of distances $s_{1}^{(j)}, s_{2}^{(j)}, \ldots s_{n}^{(j)}, \ldots$, generated by the probability density $P_{1}(s)$, characterizes the trajectory of the particle $j$ in space. Every particle follows a different characteristic path, but remains inside the large-scale magnetic topology. The probability density $P_{1}(s)$ relates the particle acceleration process to the large-scale topology. This part/aspect was never taken into account in previous acceleration models.

- The probability density $P_{2}(E)$ provides the effective electric field $E(j)_{i}$ acting on the $j$ th particle for the effective time $\tau(j)_{i}$ it spends inside the $i$ th UCS. Particles follow very complicated trajectories inside the UCS. They may be accelerated by more than one acceleration mechanisms but what actually is important for our model is the final outcome, i.e. we characterize an UCS as a simple input-output system, in which an effective DC electric field is acting. The effective action of a UCS is to increase a particle's momentum by $\Delta \boldsymbol{p}_{i}^{(j)}=e \boldsymbol{E}_{i}^{(j)} \tau_{i}^{(j)}$.

- Finally, the probability density $P_{3}(\tau)$ gives the effective time $\tau_{i}^{(j)}$ an UCS interacts with the charged particle.

The above probabilities will define the charged particle dynamics inside the flaring region. The particle $j$ starts with initial momentum $\boldsymbol{p}_{0}^{(j)}$ from the initial position $\boldsymbol{r}_{0}^{(j)}=0$ at time $t=0$. The initial momentum $\boldsymbol{p}_{0}^{(j)}$ is such that the corresponding velocity $\left|\boldsymbol{v}_{0}^{(j)}\right|$ is drawn at random from the tail of a Maxwellian, $\left|\boldsymbol{v}_{0}^{(j)}\right| \geq v_{t h}$, with $v_{t h}$ the thermal velocity. The particle is assumed to find itself in the neighbourhood of an UCS at time $t=0$, enters it immediately and undergoes a first acceleration process.

During an interaction with the UCS, the particle's momentum in principle evolves according to

$$
\boldsymbol{p}_{i+1}^{(j)}=\boldsymbol{p}_{i}^{(j)}+e \boldsymbol{E}_{i}^{(j)} \cdot \tau_{i}^{(j)},
$$

where $\boldsymbol{E}_{i}^{(j)}$ and $\tau_{i}^{(j)}$ have been generated by the corresponding probability densities $P_{2}(E)$ and $P_{3}(\tau)$.

After the particle has left the UCS, it performs a free flight until it again meets an UCS and undergoes a new acceleration process (see Fig. 29). The probability density $P_{1}(s)$ determines the spatial distance $s_{i}^{(j)}$ the particle travels before it meets this next UCS, situated at

$$
\boldsymbol{r}_{i+1}^{(j)}=\boldsymbol{r}_{i}^{(j)}+s_{i}^{(j)} \hat{r}_{i}^{(j)},
$$

where $\hat{r}_{i}^{(j)} \equiv \boldsymbol{p}_{i}^{(j)} /\left|\boldsymbol{p}_{i}^{(j)}\right|$ is a unit vector into the direction of the free flight, and $\boldsymbol{r}_{i}^{(j)}$ is the location of the previous UCS the particle had met. 


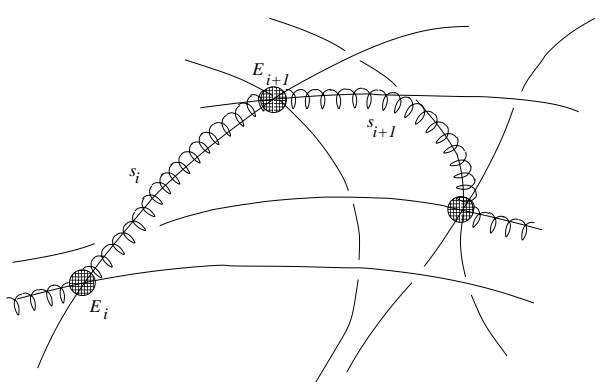

Fig. 29. Sketch of the basic elements of the model. A particle follows a magnetic field line (solid line), although undergoing drifts, and travel in this way freely a distance $s_{i}$, until enters (filled circle), where it is accelerated by the associated effective DC electric field $E_{i+1}$. After the acceleration event the particle again moves freely until it meets a new UCS. [172]

The time passed during the acceleration process and the free flight is,

$$
t_{i+1}^{(j)}=t_{i}^{(j)}+\tau_{i}^{(j)}+s_{i}^{(j)} / v_{i}^{(j)}
$$

where $t_{i+1}^{(j)}$ is the time when the particle enters the $i+1$ th UCS.

The particle starts a new cycle of acceleration and free flight at this point, the process as a whole is a cyclic one with continued probabilistic jumps in position- and momentum-space. The system was monitored for times which are relatively short, of the order of one second. For such times, the particles can be assumed to be trapped inside the overall acceleration volume $V_{a c c}=$ $L_{a c c}^{3}$, an assumption which will be confirmed by the results presented below. A second consequence of the short monitoring times is that the probability density $P_{1}(s)$, which reflects the magnetic topology and the distribution of the UCS, remains independent of time, since no large-scale changes of the topology are expected for such short times.

Let us now define the probability densities $P_{1}, P_{2}, P_{3}$ used in this study. The active, flaring region may be assumed to be in a state of MHD turbulence, embedded in a complex, large-scale magnetic topology. The UCS, i.e. the regions of dissipation, are distributed in such a way that they form in their ensemble a fractal set. This claim is based on two facts: (i) flaring active regions have successfully been modeled with Self-Organized Criticality (SOC; $[109,110,81,82])$. It was demonstrated in $[80,81,82]$ that the unstable sites in the SOC models actually represent small scale current dissipation regions, i.e. they can be considered as UCS. Furthermore, $[114,84]$ have shown that the regions of dissipation in the SOC models at fixed times form a fractal, with fractal dimension roughly $D_{F}=1.8$. (ii) From investigations of hydrodynamic turbulence we know that the eddies in the inertial regime have a scale free, power-law size distribution, making it plausible that at the dissipative scale a fractal set is formed, and indeed different experiments conclude that the 
dissipative regions form a fractal with dimension around 2.8 (see [12] and references therein).

The particles in this model are thus assumed to move from UCS to UCS, the latter being distributed such that they form a fractal set. Isliker and Vlahos [83] analyzed the kind of random walk where particles move in a volume in which a fractal resides, usually traveling freely but being scattered (accelerated) when they encounter a part of the fractal set. They showed that in this case the distribution of free-travel distances $r$ in between two subsequent encounters with the fractal is distributed in good approximation according to

$$
p(r) \propto r^{D_{F}-3}
$$

as long as $D_{F}<2$. For $D_{F}>2, p(r)$ decays exponentially. Given that a dimension $D_{F}$ below two is reported for SOC models, we are led to assume that $P_{1}(s)$ is of power-law form, with index between -1 and -3 , preferably near a value of $D_{F}-3=-1.2$ (with $D_{F}=1.8$, according to $[84,114]$ ). Not included in the study of [83] are two effects, (a) that the particles do not move on straight line paths in between two subsequent interactions with UCS, but they follow the bent magnetic field lines, and (b) that particles can be mirrored and trapped in some regions, making in this way the free travel distances larger. It is thus reasonable to consider the power-law index of $P_{1}(s)$ as a free parameter.

The freely travelled distances $s$ are distributed according to

$$
P_{1}(s)=A s^{-a}, \text { with } l_{\min }<s<l_{\max },
$$

where $l_{\min }\left(L_{a c c}\right)$ and $l_{\max }\left(L_{a c c}\right)$ are related to the characteristic length of the coronal active region $L_{a c c}$, and $A$ is a normalization constant.

The second probability density determines the effective electric field attached to a specific UCS. Its form should in principle be deduced, either from observations, which is not feasible so far, or from the simulation and modelling of a relevant set-up, which to our knowledge seems not to exist at this time. Two cases of distributions are of particular interest, the "well-behaved" case, where $P_{2}$ is Gaussian, and the "ill-behaved" case, where $P_{2}$ is of power-law form, above all with index between -1 and -3 . The Gaussian case is well behaved in the sense that all the moments are finite, and it is a reasonable choice because of the Central Limit Theorem, which suggests Gaussian distributions if the electric field is the result of the superposition of many uncontrollable, small processes. The power-law case is ill-behaved in the sense that most moments are infinite. It represents the case of scale-free processes, as they appear for instance in SOC models. A characteristic of power-law distributions is the importance of the tail, which in fact causes the dominating effects.

Trying also the case of Gaussian distributions and guided by the results, we present in this study only the case where the distribution of the electric field magnitude is of power-law form,

$$
P_{2}(E)=B E^{-b}, \text { with } E_{\min }<E<E_{\max }
$$


which shows better compatibility with the observations. We just note that most acceleration mechanisms mentioned earlier have power-law probability distributions for the driving quantity. $E_{\min }\left(E_{D}\right)$ and $E_{\max }\left(E_{D}\right)$ are related to the Dreicer field $E_{D}$, and $B$ is the normalization constant. The electric field is then determined as $\boldsymbol{E}=E \hat{\boldsymbol{r}}$, where $\hat{r}$ is a 3-D unit vector into a completely random direction.

For the distribution of acceleration times a model would also be needed. Since the acceleration times appear only in combination with the electric fields in the momentum increment, $e \boldsymbol{E}_{i}^{(j)} \cdot \tau_{i}^{(j)}$ [see Eq. 29], we can absorb any nonstandard feature, such a scale-freeness or other strong non-Gaussianities, in the distribution of $E$. This is also reasonable since both $\tau$ and $E$ are effective quantities.

It was assumed that the time a particle spends inside an UCS obeys a Gaussian distribution with mean value $t_{c}$ and standard deviation $t_{m}$,

$$
P_{3}(\tau)=C e^{-\frac{\left(\tau-t_{c}\right)^{2}}{2 t_{m}^{2}}}
$$

Defined in this way, the acceleration times are not essential for the acceleration process, they influence though the overall acceleration time-scale, i.e. the global timing of acceleration.

The simulations are performed by using $10^{6}$ particles, and the system is monitored for $1 \mathrm{sec}$, with the aim of focussing on a short time-interval during the impulsive phase (for longer times the loss of particles from the accelerating volume should be included). An extended parametric study was performed in [172]. The particles sustain repeated acceleration events, whose number differs from particle to particle: the minimum number of acceleration events per particle is found to be 1 , the maximum is 175 , and the mean is 13.4 . A substancial fraction of the particles undergoes one, initial, acceleration process. To analyze the diffusive behaviour of the particles in position space, the mean square displacement $\left\langle r^{2}(t)\right\rangle$ of the particles from the origin as a function of time was determined. For all times the system is monitored, we find strong super-diffusion, $\left\langle r^{2}(t)\right\rangle \propto t^{\gamma}$, with $\gamma$ around 3. The behaviour is different above and below $410^{-3} \mathrm{sec}$, a time which is related to the acceleration time: at $t_{c}+3 t_{m}=410^{-3} \mathrm{sec}$ the vast majority of the particles have finished their first acceleration process (since the acceleration times are Gaussian distributed [see Eq. 35], 99\% of the particles have an acceleration time smaller than the time of three standard deviations above the mean value). Below $410^{-3} \mathrm{sec}$, the particles typically are still in their first acceleration process, whereas above $410^{-3} \mathrm{sec}$, some particles are on free flights and others are in new acceleration processes. We cannot claim that the diffusive behaviour has settled to a stationary behaviour in the $1 \mathrm{sec}$ we monitor the system. At $1 \mathrm{sec}$, we find $\left\langle r^{2}(t=10)\right\rangle \approx 10^{19}=L_{\text {act }}^{2} / 10$, the particles have diffused a distance less than the active region size.

The diffusive behaviour in velocity space means determining the mean square displacement $\left\langle v^{2}(t)\right\rangle$ of the particles from their initial velocity. The 
particles start with a mean initial velocity, and after roughly $0.001 \mathrm{sec}$, a time slightly earlier than $t_{m}$, the mean acceleration time, $\left\langle v^{2}(t)\right\rangle$, starts to increase, i.e. the particles start to feel acceleration. In the range roughly from $0.001 \mathrm{~s}$ to $0.02 \mathrm{~s}$, the diffusion in energy is $\left\langle v^{2}(t)\right\rangle \propto t^{0.5}$, and in the range $0.5 \mathrm{~s}$ to 1. s, $\left\langle v^{2}(t)\right\rangle \propto t^{0.25}$. The system thus exhibits clear sub-diffusive behaviour in velocity space (the latter corresponding to the non-relativistic energy space).

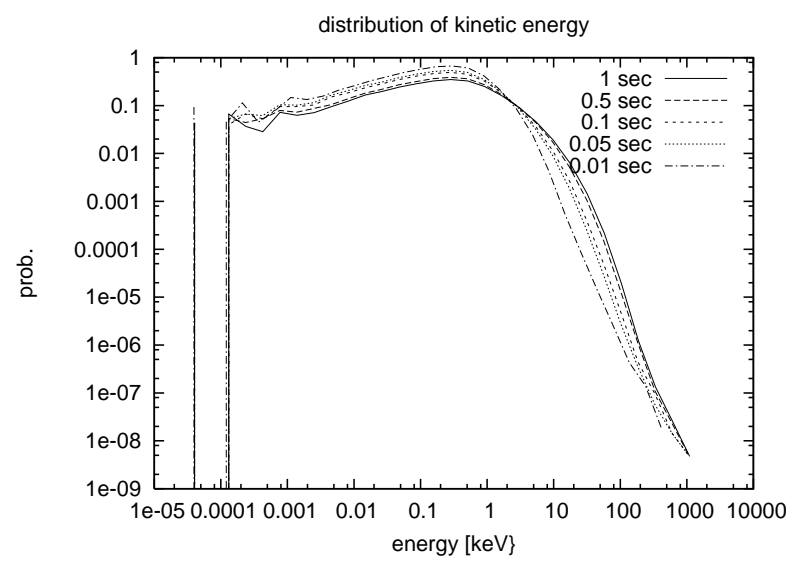

Fig. 30. Kinetic energy distributions $p\left(E_{k i n}, t\right)$ (probability density function, normalized to one) at times $t=0.01,0.05,0.1,0.5,1$, sec. [172]

At preordained times, the kinetic energy of the particles was estimated and their histograms constructed, which, normalized to 1, yield the kinetic energy distributions $p\left(E_{k i n}, t\right)$ shown in Fig. 30. The distributions retain a similar shape for the time period monitored, being flatter at low energies, and a power-law tail above roughly $5 \mathrm{keV}$. The low energy part is actually a Maxwellian. The power-law index of the high energy tail varies around 4 , increasing slightly with time, and the particles also reach higher energies with increasing time. A systematic shift of the Maxwellian towards higher energies was detected, in parallel with the development of a power-law tail that extended to higher and higher energies and steepens. At $1 \mathrm{sec}$, the most energetic particles have reached kinetic energies slightly above $1 \mathrm{MeV}$.

It is of interest to know what will happen to the ions which go through the same kind of processes. Adjusting the particle mass in the model, and keeping all the parameters fixed, the initial distribution of protons is basically unaltered, even for times up to $1000 \mathrm{sec}$. The reason is that the momentum increments are too small for the ions to undergo a visible change in distribution; they need larger momentum increments. The minimum of the electric field distribution was adjusted to $E_{\min }=100 E_{D}$, which increases the mean value of the effective electric field and so causes larger momentum increments for the protons. The energy distributions are again Maxwellians with approx- 
imate power-law tails. The index of the power-law tail is around 3.5 at small times. The Maxwellian is shifted to higher energies in the course of time, which corresponds again to heating, as in case of the electrons.

The link of the energy release processes with the acceleration of particles was studied recently in several articles [45, 40]. Dauphin [40] also uses the idea of fragmented energy release as a starting point of his acceleration model. He assumed that the majority of the particles escape from the UCS after being accelerated only once. A small fraction of the non-thermal particles interact with multiple UCS. The probability distribution function of the acceleration lengths "seen" by a particle is

$$
P(\Delta \ell)=k_{1}(\Delta \ell)^{-\delta}
$$

The idea behind this choice is that in the active region during a flare a large number of UCSs with all scales are present. The electric field distribution is similar to the one reported in Eq. 34

$$
P\left(E_{U C S}\right)=k_{2}\left(E_{U C S}\right)^{-\zeta} .
$$

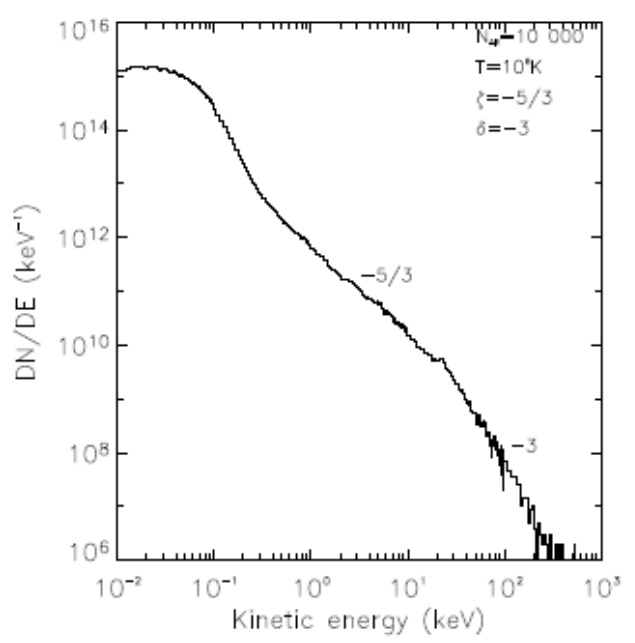

Fig. 31. Kinetic particle distribution obtained with $10000 \mathrm{UCS}, \zeta=5 / 3$ and with a distribution of particles acceleration length give by a power law spectral index $\delta=3$. The temperature of the injected distribution is $10^{6} \mathrm{~K}$. [40]

Assuming that the acceleration region is populated by thousands of UCS with different sizes distributed inside the large-scale structure, the kinetic energy distribution of the particles was estimated (see Fig. 31) and seems to agree remarkably well with the data. 


\subsection{The strongly turbulent accelerator (STA)}

In this review we have explored the evolution of particles in large-scale stressed magnetic fields, forming UCSs of different characteristic scales and at random points. Starting from the collapse of a turbulent current sheet $[52,133]$ and progressively moving to larger scales of stressed magnetic topologies [159, 160], a number of UCSs will be present during a flare or/and CME. The random appearance of UCS in the middle of a large-scale MHD structure (compact loops, eruptive structures) which host them has been studied in several articles $[63,47,48,15]$. These UCSs act as local "nodes" of a large eveolving Network [172]. These accelerators can be examined using a Monte Carlo simulation which link the energy release with the accelerator[172, 45, 40]. We name this new accelerator a Strongly Turbulent Accelerator (STA). This type of accelerator appears more frequently in magnetized strongly turbulent astrophysical systems (AGN, jets, accretion disks, galaxy clusters, black hole accretion etc). We have shown that a STA is much more efficient than most known acceleration mechanisms (shocks, MHD waves, etc) since more than $50 \%$ of the available magnetic energy of the system will end up to the high energy particles.

\section{Discussion of the global consideration of particle acceleration}

In Section 3 we suggested that there are two broad classes of flares: (1) the compact flare (closed magnetic loops) driven mainly by random photospheric flows, and (2) the eruptive structures responsible for the flare/CME driven mainly by flux emergence or unstable magnetic loops. The main theme of this review is that flares and CMEs are phenomena which are closely related with the evolution of the active region and not isolated structures. It is worth summarising the main steps followed by an active region before it reaches the state of "flaring", and the STA turns on.

1. The formation and the drivers of an active region: Newly emerged flux and photospheric motions act as the drivers of the active region complex. The nonlinear extrapolations of magnetograms remain an open problem, but force-free or simpler forms of extrapolation locate a large number of Thin Current Sheets (TCS) inside the 3-D magnetic complex $[46,107,17,18,64,14]$.

2. The large-scale 3-D magnetic topology (skeleton) hosting the flares: The 3-D magnetic structures (compact loops or eruptive structures) form the skeleton in which the explosive phenomena (flares, CMEs) take place.

3. Storage of magnetic energy inside the large-scale structure: There is the formation of large QSL or thin current sheets (TCS) that are sta- 
ble structures and can store magnetic energy occurs. The energy can be released if the resistivity exceeds a certain value $[171,36]$.

4. The trigger of explosions: The continuous stresses from the driver or the emergence of new magnetic flux forces some TCSs (sometimes even one with very large scale may be enough) to become unstable (a UCS). The appearance of one or more UCS will cause a catastrophic collapse of many current sheets $[36,133]$.

5. The formation of a turbulent active region: The appearance of many UCS in the 3-D large-scale structure forms a strongly turbulent active region with many localized UCS. In some flares the appearance of UCSs may start before any sudden eruption, and there is continued release of energy in repeated explosions for 100s of seconds after the first major eruption.

6. The acceleration of particles inside a strongly turbulent active region: The particle evolution inside a strongly turbulent active region is a very fast and efficient accelerator with many UCS acting as nodes of acceleration. This accelerator is called a STA [52, 133, 159, 160, 15, 47, $48,172,45,40]$.

Miller et al. [122] suggested that a model can be a viable particle accelerator for flares, if it has a number of properties based on the observational constrains existing in the mid 1990s. We now assess the ability of the STA to follow these constrains.

- Can a STA accelerate electrons up to $100 \mathrm{keV}$ (responsible for HXR)? All UCS, even when isolated, with sizes larger that $100 \mathrm{~s}$ of $\mathrm{km}$ can accelerate electrons up to $100 \mathrm{keV}[152,127,106,179,180,41,42,52$, 133, 89].

- Can a STA accelerate electrons with energies of 10s of MeV? Electrons interacting with several UCS can reach very high energies. When interacting with a single UCS, it is difficult to reach these energies, but a network of UCS will accelerate electrons up to $10 \mathrm{~s}$ of $\mathrm{MeV}$ and ions up to GeV. [160, 40, 172]

- Can the STA accelerate ions? Yes, very efficiently [160, 40, 172].

- Is the STA a fast accelerator (secs)? Most of the studies done today suggest that it is extremely fast (less than a second) [52, 133, 159, 160, 15, $47,48,172]$.

- Does the STA reproduce the observed electron distribution? The models proposed above, either a single collapsing UCS, and especially the network of UCS can reproduce the generic distribution function for electrons $[52,133,160,40,172,47]$.

- Does the STA reproduce the observed proton distribution? The models proposed above, either the collapsing single UCS or the network of UCS can reproduce the distribution function for protons [160, 172].

- Does the STA produce a heavy ion enhancement? It is not clear yet how this can happen. 
- Can the STA accelerate electrons and ions from thermal particles? Yes the UCSs can accelerate electrons and ions from the thermal background [160, 172, 40, 47, 52, 133].

- Is filamentation and complexity essential for the STA? Yes both are essential for this model.

- Is the mechanism well connected with the global structure? The STA is based on the energy release (UCS) and the evolution of large-scale structures.

Let us now add to the observational constrains known ten years ago those reported in Section 2 based on the recent data collected from RHESSI.

1. The number problem? The fact that UCS are distributed along the large-scale structures will be very helpful. One important factor, still unexplored, is the fact that UCS can be present even in the foot points of the large-scale structures. The competition between acceleration and collisions will make the number problem less severe. This is an open problem and needs more careful analysis.

2. Energetics? The STA is extremely efficient accelerator $[52,133]$.

3. Flare statistics? The turbulent flare, on which the STA is based, is well connected to Self-Organized Criticality theory [172, 40].

4. Temporal evolution of the Power law index? Still an open problem.

5. Coronal X-ray sources? Particle diffusion inside the STA is not normal, and particles undergo many interactions with the UCSs, therefore their diffusion along the field lines slows down dramatically. No detailed estimates of the confinement of particles in the turbulent corona are yet available.

6. The close temporal and spectral evolution of the foot-points? The fact that UCSs are distributed along evolving largescale structure suggest that the STA can reproduce this result but more work is needed.

7. The close correlation of HXR with particles detected in space? This is closely related to the large-scale topology and the position of the UCS. In principle many simple 3-D topologies can allow this coincidence to exist.

\section{Summary}

In this review we have noted the most striking new observational constrains for particle acceleration during solar flares and linked the flare energy release with the acceleration. Before constructing a model for particle acceleration in the Sun we have to understand the formation and the energy release processes in active regions.

We have shown that driven active regions form thin current sheets (TCS). Large-scale structures are stressed beyond a certain point and one or several TCSs become suddenly Unstable Current Sheets (UCS). The UCS collapse 
and force several other stable TCSs to become UCS (an avalanche). This is the way a turbulent flare will begin inside the large-scale structure of an active region.

Acceleration of particles inside many unstable collapsing UCS has been discussed by many authors, and a new acceleration processes has been emerged, the Strongly Turbulent Accelerator (STA). The STA can easily explain most of constraints reported from the recent data.

There are three major theoretical challenges:

1. The nonlinear extrapolation of observed magnetograms and the detailed formation of the TCS remains an open and challenging problem.

2. Although the evolution of large-scale structures can be analyzed easily with 3-D MHD codes, the evolution of unstable current sheets should be analyzed with 3-D kinetic codes. No current code can handle the simultaneous presence of so many scales.

3. The strange kinetics, and the anomalous diffusion of the accelerated particles inside a turbulent flare (an evolving network of UCS's) does not follow the Boltzmann equation. Forming fractional kinetic equations for the evolution of particles in strongly turbulent plasmas is not an easy task.

The main observational challenge is the direct observation of the fragmentation of the energy release. Large spatial and temporal resolution is needed and the emphasis should be shifted to smaller flares and their statistical properties. The Ellerman bombs is an example of "micro flaring" [66] with many interesting points along the lines discussed here.

Acknowledgement. We would like to thank our colleagues Drs A. Anastasiadis, K. Arzner, H. Isliker, K. Galsgaard, M. Georgoulis, F. Lebreti, M. Onofri, R. Turkmani and Mr T. Fragos for many useful conversations.

\section{References}

1. V.I. Abramenko, V. Yurchyshyn, H. Wang, P.R. Goode: Solar Phys., 201, 225 (2001)

2. V.I. Abramenko, V. Yurchyshyn, H. Wang, T.J. Spirock, P.R. Goode: Astrophys. J., 577, 487 (2002)

3. V.I. Abramenko, V. Yurchyshyn, H. Wang, T.J. Spirock, P.R. Goode: Astrophys. J., 597, 1135 (2003)

4. A. Achterberg: Astron. Astrophys., 97, 259 (1981)

5. R. Albert and A.L. Barabasi: Rev. Modern Phys., 47, 47 (2002)

6. C.E. Alissandrakis: Astron. Astrophys., 100, 197 (1981)

7. T. Amari, J.F. Luciani, J.J. Aly, Z. Mikic, J. Linker: Astrophys. J., 595, 1231 (2003)

8. J. Ambrosiano, W.H. Matthaeus, M.L. Goldstein, and D. Plante: J. Geophys. Res., 93, 14383 (1988) 
9. A. Anastasiadis and L. Vlahos: Astron. Astrophys., 245, 271 (1991)

10. A. Anastasiadis and L. Vlahos: Astrophys. J., 428, 819 (1994)

11. A. Anastasiadis, L. Vlahos, M. Georgoulis: Astrophys. J., 489, 367 (1997)

12. F. Anselmet, R.A. Antonia, L. Danaila: Planetary and Space Science 49, 1177 (2001)

13. S. Antiochos, C.R. DeVore, J.A. Klimchuk: Astrophys. J., 510, 485 (1999)

14. V. Archontis, A.W. Hood, C. Brady: Astron. Astrophys., 466, 367 (2007)

15. K. Arzner and L. Vlahos: Astrophys. J. Lett, 605, L69 (2004)

16. M.J. Aschwanden, T. Kosugi, H.S. Hudson, M.J. Wills, R.A. Schwartz: Astrophys. J., 470, 1198 (1996)

17. G. Aulanier, E. Pariat, P. Demoulin: Astron. Astrophys., 444, 961 (2005)

18. G. Aulanier, E. Pariat, P. Demoulin: Solar Phys., 238, 347 (2006)

19. P. Bak, C. Tang, K. Wiesenfeld: 1987, Phys. Rev. Lett., 59, 381 (1987)

20. P. Bak: How Nature Works, New York: Springer Verlag (1996)

21. R. Balescu: Statistical Dynamics: Matter out of Equilibrium, Imperial College Press (1997)

22. S.G. Benka and G.D. Holman: Astrophys. J., 391, 854 (1992)

23. A.O. Benz: Space Science Rev., 68, 135 (1994)

24. A.O. Benz: in Energy Conversion and Particle Acceleration in the Solar Corona, ed. K.L. Klein (Springer, Berlin), p. 80 (2003)

25. D. Biskamp: Magnetohydrodynamic turbulence, Campbridge University Press (2003)

26. D. Biskamp: Magnetic Reconnection in plasmas, Cambridge University Press (2000)

27. R. Blandford and D. Eichler: Phys. Rep., 154, 1, (1987)

28. D. Borgogno et al.: Phys. Plasmas, 12, 32309 (2005)

29. J.L. Bougeret et al.: Space Sci. Rev. 71, 231 (1995)

30. J.C. Brown: Solar Phys., 18, 489, (1971)

31. J.C. Brown, A.G. Emslie, E.P. Kontor: Astrophys. J. Lett., 595, L115 (2003)

32. A.C. Cadavid, J.K. Lawrence, A.A. Ruzmaikin, A. Kayleng-Knight: Astrophys. J., 429, 391 (1994)

33. P.J. Cargill and E.R. Priest: Astrophys. J., 266, 383 (1983)

34. P.J. Cargill: in SOLMAG: Magnetic coupling of the solar atmosphere, ESA SP505, p. 245 (2002)

35. P.J. Cargill, L. Vlahos, R. Turkmani, K. Galsgaard, H.Isliker: Space Sci. Rev., 124, $246(2006)$

36. P.A. Cassak, M.A. Shay, J.F. Drake: Phys. Rev. Lett., 95, 235002 (2005)

37. S. Chandrasekhar: Rev. Mod. Phys., 15, 1 (1943)

38. N.B. Crosby, M.J. Aschwanden, B.R. Dennis: Solar Phys. 143, 275 (1993) ,

39. S. Daikou et al.: Astrophys. J., 634, 663 (2005)

40. C. Dauphin: Astron. Astrophys., 471, 993 (2007)

41. S. Dalla and P.K. Browning: Astron. Astrophys., 436, 1103 (2005)

42. S. Dalla and P.K. Browning: Astrophys. J. Lett., 640, L99 (2006)

43. R.B. Decker and L. Vlahos: Astrophys. J., 306, 710 (1986)

44. R.B. Decker: Space Sci. Rev. 48, 195 (1988)

45. N. Décamp and F. Malara: Astrophys. J. Lett., 637, L61 (2006)

46. P. Démoulin: Adv. Space Res., 39, 1367 (2007)

47. P. Dmitruk, W.H. Matthaeus, N. Seenu, M.R. Brown: Astrophys. J. Lett., 597, L81 (2003) 
48. P. Dmitruk, W.H. Matthaeus, N. Seenu: Astrophys. J., 617, 667 (2004)

49. S.N. Dorogovsky, J.F. Mendes, A.N. Samuhhin: Phys. Rev. Letters 85, 4633 (2000)

50. S.N. Dorogovtsev and J.F. Mendes: Adv. in Phys. 51, 1079 (2002)

51. J.F. Drake, M.A. Shay, W. Thongthai, M. Swisdak: Phys. Rev. Lett., 94, 95001 (2005)

52. J.F. Drake, M. Swisdak, H. Che, M.A. Shay: Nature, 443, 553 (2006)

53. J.F. Drake, M. Swisdak, K.M. Schoeffler, B.N. Rogers, S. Kobayashi: J. Geophys. Res., 33, L13105 (2006)

54. C. Efthimiopoulos, C. Gontikakis, A. Anastasiadis: Astron. Astrophys., 443, $643(2005)$

55. D.C. Ellison and R. Ramaty: Astrophys. J., 298, 400 (1985)

56. A.G. Emslie et al.: J. Geophys. Res., 109, A10104 (2004)

57. A.G. Emslie, B.R. Dennis, G.D. Holman, H.S. Hudson: J. Geophys. Res., 110, A11103 (2005)

58. E. Fermi: Phys. Rev., 75, 1169 (1949)

59. L. Fletcher and H.S. Hudson: Solar Phys., 210, 307 (2002)

60. T.G. Forbes and E.R. Priest: Astrophys. J., 446, 377 (1995)

61. T. Fragos, M. Rantziou, L. Vlahos: Astron. Astrophys., (2003)

62. K. Galsgaard and A. Nordlund: J. Geophys. Res., 101, 13445 (1996)

63. K. Galsgaard: in SOLMAG: Magnetic Coupling of the Solar Atmosphere, ESA SP-505 (2002)

64. K. Galsgaard, V. Archontis, F. Moreno-Insertis, A.W. Hood: Astrophys. J., 666, 516 (2007)

65. M. K. Georgoulis, B.J. LaBonte, T.R. Metcalf: Astrophys. J., 602, 447 (2004)

66. M.K. Georgoulis, D.M. Rust, P.N. Bernasconi, B. Schmieder: Astrophys. J., $\mathbf{5 7 5}, 506(2002)$

67. M.K. Georgoulis and B.J. LaBonte: Astrophys. J., 615, 1029 (2004)

68. M.K. Georgoulis: Solar Phys., 228, 5 (2005)

69. H. Gould and J. Tobochnik: An Introduction to Computer Methods, 2nd Edition, Adison-Wesley (1996)

70. P.C. Grigis and A.O. Benz: Astron. Astrophys., 426, 1093 (2004)

71. P. C. Grigis and A.O. Benz: Astron. Astrophys., 434, 1173 (2005)

72. K.L. Harvey: PhD thesis, University of Utrecht, Netherlands (1993)

73. K. L. Harvey and C. Zwaan: Solar Phys., 148, 85 (1993)

74. J. Heyvaerts: in Solar Flare Magnetohydrodynamics, ed E.R. Priest (New York: Gordon and Beach), p. 429 (1981)

75. G.D. Holman and M.E. Pesses: Astrophys. J. 267, 837 (1983)

76. R. F. Howard: Ann. Rev. Astron. Astrophys., 34, 75 (1996)

77. D. Hughes and M. Paczuski: Phys. Rev. Lett., 88, 054302 (2002)

78. D. Hughes, M. Paczuski, R.O. Dendy, P. Halander, K.G. McClements: Phys. Rev. Lett., 90, 131 (2003)

79. G.J. Hurford, R.A. Schwartz, S. Krucker, R.P. Lin, D.M. Smith, N. Vilmer: Astrophys. J. Lett., 595, L77 (2003)

80. H. Isliker, A. Anastasiadis, D. Vassiliadis, L. Vlahos: Astron. Astrophys., 363, 1134 (1998)

81. H. Isliker, A. Anastasiadis, L. Vlahos: Astron. Astrophys., 363, 1134 (2000)

82. H. Isliker, A. Anastasiadis, L. Vlahos: Astron. Astrophys., 377, 1068 (2001)

83. H. Isliker and L. Vlahos: Phys. Rev. E, 67, 026413 (2003) 
84. H. Isliker and L. Vlahos: unpublished result (2003)

85. H. Ji et al.: Phys. Rev. Lett., 92, 115001 (2004)

86. A.L. Kiplinger: Astrophys. J., 453, 973 (1995)

87. J.G. Kirk: Plasma Astrophysics (Springer, Berlin), p. 225 (1994)

88. B. Kliem: Astrophys. J., 90, 719, (1994)

89. B. Kliem, M. Karlicky, A.O. Benz: Astron. Astrophys., 360, 715 (2000)

90. R.A. Kopp and G.W. Pneuman: Solar Phys., 50, 85 (1976)

91. S. Krucker and R.P. Lin: Solar Phys., 210, 229 (2002)

92. S. Krucker, M.D. Fivian, R.P. Lin: Adv. Space Res., 35(10), 1707 (2005)

93. S. Krucker, G.J. Hurford, R.P. Lin: Astrophys. J. Lett., 595, L103 (2003)

94. S. Krucker and R.P. Lin: Astrophys. J. Lett., 542, L61 (2000)

95. J. Kuijpers: Lect. Notes Phys. 469, 101 (1996)

96. R.M. Kulsrud and A. Ferrari: Astrophys. and Space Sci., 12, 302 (1971)

97. P. Langevin: C.R. Acad. Sci. (Paris), 146, 530 (1908)

98. T.N. Larosa and R.L. Moore: Astrophys. J., 418, 912 (1993)

99. Y.T. Lau: Solar Phys., 148, 301 (1993)

100. J.K. Lawrence, A.A. Ruzmaikin, A.C. Cadavid: Astrophys. J., 417, 805 (1993)

101. J.K. Lawrence, A.C. Cadavid, A.A. Ruzmaikin: Astrophys. J., 465, 425 (1996)

102. K.D. Leka and A. Skumanich: Solar Phys., 188, 3 (1999)

103. A.J. Lichtenberg and M.A. Lieberman: Regular and Stchastic motion, SpringerVerlag (1983)

104. R.P. Lin and the RHESSI Team: Proc. 10th European Solar Physics Meeting, Prague (ESA SP-506, 2002), p. 1035 (2002)

105. R.P. Lin et al.: Astrophys. J. Lett., 595, L69 (2003)

106. Y.E. Litvinenko: Solar Phys., 212, 379 (2003)

107. D.W. Longcope: Living Rev. Solar Phys. 2, 7 (2005)

108. D. W. Longcope and H.R. Strauss: Astrophys. J., 437, 851 (1994)

109. E.T. Lu and R.J. Hamilton: Astrophys. J. Lett., 380, L89. (1991)

110. E.T. Lu, R.J. Hamilton, J.M. McTiernan, K.R. Bromund: Astrophys. J., 412, 841 (1993)

111. P.C.H. Martens: Astrophys. J. Lett., 330, L131 (1988)

112. S. Masuda, T. Kosugi, H. Hara, S. Tsuneta, Y. Ogawara: Nature 371, 595 (1994)

113. A.N. McClynmont, L. Jiao, Z. Mikic: Solar Phys., 174, 191 (1997)

114. S.W. McIntosh, P. Charbonneau, J.P. Norman, T.J. Bogdan, H.L. Liu: Phys. Rev. E, 65, 46125 (2002)

115. K. P. Macpherson and A.L. MacKinnon: Astron. Astrophys. 350, 1040 (1999)

116. D.B. Melrose: in Particle acceleration in cosmic plasmas, ed. G. P. Zank and T.K. Gaisser (New York: Institute of Physics), p. 3 (1992)

117. N. Meunier: Astrophys. J., 515, 801 (1999)

118. N. Meunier: Astron. Astrophys., 420, 333 (2004)

119. L.J. Milano, P. Dmitruk, C.H. Mandrini, D.O. Gómez, P. Demoulin: Astrophys. J., 521, 889 (1999)

120. J.A. Miller and D.A. Roberts: Astrophys. J., 452, 912 (1995)

121. J.A. Miller, T.N. LaRosa, R.L. Moore: Astrophys. J., 461, 445 (1996)

122. J.A. Miller, P.J. Cargill et al.: J. Geophys. Res., 102, 14631 (1997)

123. R. Metzler and J. Klafter: Phys. Rep., 339, 1 (2000)

124. E.L. Moghaddam-Taaheri, L. Vlahos, H.L. Rowland, K. Papadopoulos: Phys. Fluids, 28, 3356 (1985) 
125. E.L. Moghaddam-Taaheri and C. K. Goertz: Astrophys. J., 352, 361 (1990)

126. M.E.J. Newman and G.T. Barkema: Monte Carlo Methods in Statistical Physics, Oxford University Press (2001)

127. C. Nodes, G.T. Birk, H. Leach, R. Schopper: Phys. Plasmas, 10, 835 (2003)

128. A. Nordlund and K. Galsgaard: in Solar and heliospheric plasma physics, Eds G.M. Simnett, C.A. Allisandrakis \& L. Vlahos, Springer Verlag (Berlin) (1996)

129. D.R. Nicholson: Introduction to plasma theory, J. Wiley and Sons (New York) (1983)

130. G. Nicolis and I. Prigogin: Exploring Complexity: An introduction, New York: Freeman Co. (1989)

131. M. Øieroset, R.P. Lin, T.D. Phan, D.E. Larson, S.D. Bale: Phys. Rev. Lett., 89, $195001(2002)$

132. M. Onofri, L. Primavera, F. Malara, P. Veltri, Phys. Plasmas, 11, 4837

133. M. Onofri, H. Isliker, L. Vlahos: Phys. Rev. Lett., 96, 151102 (2006)

134. K. Papadopoulos: Rev. Geophys. Space Phys., 15, 113 (1977)

135. E.N. Parker: Astrophys. J. 174, 642 (1972)

136. E.N. Parker: Astrophys. J., 264, 642 (1983)

137. E.N. Parker: Astrophys. J. 330, 474 (1988)

138. C.E. Parnell and P.E. Jupp: Astrophys. J., 529, 554 (2000)

139. V. Petrosian and T. Donaghy: Astrophys. J., 610, 550 (1999)

140. V. Petrosian, H. Yan, A. Lazarian: Astrophys. J., 644, 603 (2006)

141. H.E. Petschek: in The physics of solar flares (ed.: Hess, W. N.), Proc. AASNASA Symposium, SP-50 p. 425 (1964)

142. E.R. Priest and T.G. Forbes: Magnetic Reconnection: MHD theory and applications, Cambridge University Press (2000)

143. E.R. Priest and T.G. Forbes: Astr \& Astrophys. Rev., 10, 313 (2002)

144. E.R. Priest, G. Hornig, D.I. Pontin: J. Geophys. Res., 108, 1285 (2003)

145. P.L. Prietchett: J. Geophys. Res., 106, 3783 (2001)

146. J. Qiu, J. Lee, D.E. Gary, H. Wang: Astrophys. J., 565, 1335 (2002)

147. H. Risken: The Fokker-Planck Equation, 2nd Ed., Berlin, Springer (1989)

148. I.I. Rousev et al: Astrophys. J. Lett., 588, L45 (2003)

149. P. Saint-Hilaire and A.O. Benz: Solar Phys., 210, 287 (2002)

150. T. Sakao, T. Kosugi, S. Masuda: in Observational Plasma Astrophysics: Five Years of Yohkoh and Beyond, T. Watanabe, T. Kosugi, and A.C. Sterling (ed.), , Boston, Mass., Kluwer Academic Publishers, ASSL 229, 273 (1998)

151. P.E. Seiden and D.G. Wentzel: Astrophys. J., 460, 522 (1996)

152. R. Schopper, G.T. Birk, H Lesch: Phys. Plasmas, 6, 4318 (1999)

153. K. Shibata et al.: Astrophys. J. Lett., 451, L83 (1995)

154. K. Shibata and S. Tanima: Earth Planets Space, 53, 473 (2001)

155. D.M. Smith, G.H. Share, R.J. Murphy, R.A. Schwartz, A.Y. Shih, R.P. Lin: Astrophys. J. Lett., 595, L81 (2003)

156. L. Sui and G.D. Holman: Astrophys. J. Lett., 596, L251 (2003)

157. L. Sui, G.D. Holman, B.R. Dennis, S. Krucker, R.A. Schwartz, K. Tolbert: Solar Phys., 210, 245 (2002)

158. V.S. Titov, E.R. Priest, P. Démoulin: Astron. Astrophys., 276, 564 (1993)

159. R. Turkmani, L. Vlahos, P.J. Cargill, K. Galsgaard, H. Isliker: Astrophys. J. Lett., 620, L59 (2005)

160. R. Turkmani, P.J. Cargill, K. Galsgaard, L. Vlahos, H. Isliker: Astron. Astrophys., 449, 749 (2006) 
161. B.A. Tverskoi: Soviet Phys.-JEPT Lett., 25, 317 (1967)

162. A. M. Veronig, J.C. Brown, B.R. Dennis, R.A. Schwartz, L. Sui, A.K. Tolbert: Astrophys. J., 621, 482 (2005)

163. B. Viticchié, D. DelMoro, F. Berrilli: Astrophys. J., 652, 1734 (2006)

164. L. Vlahos et al.: in Energetic phenomena on the Sun, Eds. Kundu, M., Woodgate, B., NASA Conference Publication 2439 (1986)

165. L. Vlahos: in Statistical Description of Transport in Plasmas, Astro- and Nuclear Physics, Eds J. Misquich, G. Pelletier, P. Schuck, Nova Science Publishers Inc. (New York) (1993)

166. L. Vlahos: Space Sci. Rev., 68, 39 (1994)

167. L. Vlahos, M. Georgoulis, R. Kluiving, P. Paschos: Astron. Astrophys., 299, $897(1995)$

168. L. Vlahos: in Radio emission from the stars and the sun, Eds. Taylor, A.R. \& Paredes, J.M., ASP Conference Series, 93, ASP press, San Francisco, p. 355. (1996)

169. L. Vlahos: ESA Publications, SP-505, 105 (2002)

170. L. Vlahos, T. Fragos, H. Isliker, M. Gergoulis: Astrophys. J. Lett.,, 575, L87. (2002)

171. L. Vlahos and M. Georgoulis: Astrophys. J. Lett., 603, L61 (2004)

172. L. Vlahos, H. Isliker, F. Lepreti: Astrophys. J., 608, 540 (2005)

173. L. Vlahos: in The high Energy solar corona: Waves, Eruptions, Particles, L. Klein and MacKinon, A.L. (eds) Lecture notes in Physics, p. 15. (2007)

174. D.G. Wentzel and P.E. Seiden: Astrophys. J., 390, 280 (1992)

175. T. Yokoyama, K. Shibata: Astrophys. J., 549, 1160 (2001)

176. G.M. Zaslavsky: Phys. Report, 731, 461 (2002)

177. Zeiler et al.: J. Geophys. Res., 107, 1230 (2002)

178. L. M. Zelenyi, A.V. Milanov, G. Zimabrdo: in Multiscale magnetic structures in the daistant tail, ed. A. Nishida, D.N. Baker and S.N. Cowley (American Geophysical Union, Washington DC, p. 37 (1998)

179. V. Zharkova and M. Gordovsky: Astrophys. J., 604, 884 (2005)

180. V. Zharkova and M. Gordovsky: MNRAS, 356, 1107 (2005)

181. H. Zirin: Astrophysics of the Sun, Cambridge University Press, Cambridge (1988) 